\title{
Rho-associated Protein Kinase 2 Confers Epileptogenesis through the Activation of Astroglial Stat3 Pathway
}

\author{
Li-jia Song \\ Fourth Military Medical University: Air Force Medical University \\ Hua Zhang \\ Xi'an Jiaotong University \\ Jun-gong Jin \\ International Medical Center \\ Chao Wang \\ Fourth Military Medical University: Air Force Medical University \\ Xiao-Peng Qu \\ Fourth Military Medical University: Air Force Medical University \\ Xue Jiang \\ Fourth Military Medical University: Air Force Medical University \\ Li Gao \\ Fourth Military Medical University: Air Force Medical University \\ Gang Li \\ Fourth Military Medical University: Air Force Medical University \\ Da-li Wang \\ Fourth Military Medical University: Air Force Medical University \\ Liang-liang Shen \\ Fourth Military Medical University: Air Force Medical University \\ bei liu ( $\square$ liubei206@163.com) \\ Fourth Military Medical University: Air Force Medical University
}

\section{Research}

Keywords: epilepsy, hippocampal sclerosis, epileptogenesis, ROCK2, Stat3

Posted Date: December 1st, 2020

DOI: https://doi.org/10.21203/rs.3.rs-115889/v1

License: (c) This work is licensed under a Creative Commons Attribution 4.0 International License. Read Full License 


\section{Abstract}

Patients with temporal lobe epilepsy (TLE) are prone to tolerance to antiepileptic drugs. Based on the perspective of molecular targets for drug resistance, it is necessary to explore effective drug resistant genes and signaling pathways for the treatment of TLE. We performed gene expression profiles in hippocampus of patients with drug-resistant TLE and identified ROCK2 as one of the 20 most significantly increased genes in hippocampus. In vitro and in vivo experiments were performed to identify the potential role of ROCK2 in epileptogenesis. In addition, the activity of Stat3 pathway was tested in hippocampal tissues and primary cultured astrocytes. The expression levels of ROCK2 in the hippocampus of TLE patients were significantly increased compared with the control group, which was due to the hypomethylation of ROCK2 promoter. Fasudil, a specific Rho-kinase inhibitor, alleviated epileptic seizures in the pilocarpine rat model of TLE. Furthermore, ROCK2 activated the Stat3 pathway in pilocarpine-treated epilepsy rats, and the spearman correlation method confirmed that ROCK2 is associated with Stat3 activation in TLE patients. In addition, ROCK2 was predominantly expressed in astrocytes during epileptogenesis, and induced epileptogenesis by activating astrocyte cell cycle progression via Stat3 pathway. The overexpressed ROCK2 plays an important role in the pathogenesis of drug-resistant epilepsy. ROCK2 accelerates astrocytes cell cycle progression via the activation of Stat3 pathway likely provides the key to explaining the process of epileptogenesis.

\section{Introduction}

The Rho GTPases belong to the Ras superfamily of 20 to $30 \mathrm{kDa}$ GTP-binding proteins that have been involved in the regulation of a broad spectrum of cellular functions ${ }^{1}$. Rho activation inhibits neurite outgrowth and promotes growth cone collapse and axon retraction ${ }^{2}$. RhoA is a member of the Rho GTPases and is abundantly expressed in cells of the CNS ${ }^{3}$. Recent research indicated that kainic acid-induced seizures result in bilateral RhoA activation in the cortex and hippocampus ${ }^{4}$.

ROCK, one of the most studied downstream effectors of RhoA, is a serine/threonine kinase of $160 \mathrm{kDa}$ that exists in two isoforms in mammals: ROCK1 and ROCK $2{ }^{5}$. Both ROCK1 and ROCK2 are widely expressed in human, rat and mouse tissues ${ }^{6-8}$. ROCK1 is more prominent in liver, testes and kidney, whereas ROCK2 is more pronounced in brain ${ }^{5}$. Y-27632 and Fasudil are known as specific Rho-kinase inhibitors and are clinically used to treat cerebral vasospasm following subarachnoid hemorrhage ${ }^{9}$.

ROCK2 immunoreactivity was observed in the pyramidal neurons of the cerebral cortex and hippocampus and in the Purkinje cells of the cerebellum ${ }^{10}$. ROCK activation is necessary for neurite retraction in the injured CNS ${ }^{11}$. Additionally, ROCK acts to reduce the degree of plasticity at hippocampal synapses during long-term potentiation ${ }^{12}$. Rho/ROCK pathway plays an important role in hippocampus-dependent long-term spatial memory ${ }^{13}$, and is even considered as a potential drug target in various neurological disorders ${ }^{14}$.

Recently, it has been reported that Rho/ROCK pathway plays a role in epilepsy induced by PTZ or MES ${ }^{15}$, and directly involved in seizure-induced cell death in vivo and in vitro ${ }^{16}$. In this study, we aimed to determine the expression and cellular distribution of ROCK2 in epilepsy and investigate the efficacy of Fasudil in lithium-pilocarpine induced rat model of epilepsy. Furthermore, we demonstrated that ROCK2 induces gliosis via activating Stat3 target gene Myc and Cyclin D1. Together, these observations showed that ROCK2 induces the development of epilepsy, potentially through the activation of astrocyte cell cycle progression via Stat3 pathway.

\section{Methods}

\section{Human tissue specimens}

All patients were diagnosed with TLE based on having recurrent epilepsy and being refractory to the maximal doses of at least 3 AEDs. All patients with mesial TLE with hippocampal sclerosis were treated surgically in Department of Neurosurgery, Tangdu Hospital, Fourth Military Medical University (Fig 2A.). All hippocampal specimens were randomly selected from an epilepsy brain bank. The criteria, informed consent, brain tissue processing, and how to match control groups were referenced in our previous publications ${ }^{17-19}$. The individual patient details are listed in Tables 1 and 2. 
Table 1

Clinical characteristics of TLE patients

\begin{tabular}{|c|c|c|c|c|c|c|c|c|c|c|}
\hline No & $\operatorname{Age}(y) / \operatorname{sex}$ & $S F(/ w)$ & $\begin{array}{l}\text { Course } \\
\text { (y) }\end{array}$ & $\begin{array}{l}\text { Seizure } \\
\text { type }\end{array}$ & AEDs before surgery & $\begin{array}{l}\text { Surgical } \\
\text { approach }\end{array}$ & Pathology & $\begin{array}{l}\text { Follow- } \\
\text { Up } \\
\text { duration } \\
\text { (v) }\end{array}$ & $\begin{array}{l}\text { Surgical } \\
\text { Outcome }\end{array}$ & Application \\
\hline 1 & 19/M & $1-2$ & 14 & CPS & VPA, LEV, CZP & LTHAR & FCD,g & 5 & 1 & $\begin{array}{l}\text { GXP, WB, } \\
\text { IHC, MSP }\end{array}$ \\
\hline 2 & $16 / F$ & 1 & 15 & CPS,SGS & VPA, OXC, LEV,LTG & LTHAR & $\mathrm{g}$ & 7 & 1 & $\begin{array}{l}\text { GXP, WB, } \\
\text { IHC, MSP }\end{array}$ \\
\hline 3 & $33 / \mathrm{M}$ & $2-3$ & 17 & CPS & LEV,TPM,OXC, CZP & LTHAR & $\mathrm{g}$ & 6 & 1 & $\begin{array}{l}\text { GXP, WB, } \\
\text { IHC, MSP }\end{array}$ \\
\hline 4 & $25 / \mathrm{M}$ & $1-2$ & 23 & CPS,SGS & VPA, LEV, CBZ, CZP & LTHAR & $\mathrm{g}, \mathrm{nl}$ & 9 & 1 & $\begin{array}{l}\text { GXP, WB, } \\
\text { IHC, MSP }\end{array}$ \\
\hline 5 & $12 / \mathrm{M}$ & $3-5$ & 8 & CPS & VPA,PHT,CBZ,LTG,LEV & LTHAR & FCD,g & 10 & 1 & $\begin{array}{l}\text { GXP, WB, } \\
\text { IHC, MSP }\end{array}$ \\
\hline 6 & $15 / F$ & 2 & 11 & CPS & VPA,OXC,LTG & LTHAR & FCD,g & 6 & 1 & $\begin{array}{l}\text { GXP, WB, } \\
\text { IHC, MSP }\end{array}$ \\
\hline 7 & $9 / F$ & $2-3$ & 9 & CPS,SGS & OXC, LEV, LTG & LTHAR & $\mathrm{g}, \mathrm{nl}$ & 8 & 1 & $\begin{array}{l}\text { GXP, WB, } \\
\text { IHC, MSP }\end{array}$ \\
\hline 8 & $24 / \mathrm{M}$ & $4-5$ & 9 & CPS,SGS & VPA,OXC,LEV,LTG,CBZ & LTHAR & $\mathrm{FCD}, \mathrm{g}$ & 6 & 1 & $\begin{array}{l}\text { GXP, WB, } \\
\text { IHC, MSP }\end{array}$ \\
\hline 9 & $7 / F$ & 7 & 4 & CPS & VPA,OXC,LEV & RTHAR & FCD,g & 11 & 2 & $\begin{array}{l}\text { WB, IHC, } \\
\text { MSP }\end{array}$ \\
\hline 10 & $18 / \mathrm{M}$ & $3-4$ & 7 & CPS & VPA,LEV,LTG,OXC & RTHAR & $\mathrm{g}$ & 5 & 1 & $\begin{array}{l}\text { WB, IHC, } \\
\text { MSP }\end{array}$ \\
\hline 11 & $27 / M$ & $1-2$ & 13 & CPS & VPA,CBZ,PHT,LEV,LTG & LTHAR & FCD,g,nl & 3.5 & 1 & $\begin{array}{l}\text { WB, IHC, } \\
\text { MSP }\end{array}$ \\
\hline 12 & $24 / F$ & $2-3$ & 21 & CPS,SGS & VPA,LEV,OXC,LTG & LTHAR & $\mathrm{g}$ & 9 & 3 & $\begin{array}{l}\text { WB, IHC, } \\
\text { MSP }\end{array}$ \\
\hline 13 & $42 / F$ & $1-3$ & 27 & CPS & VPA,LEV,OXC,LTG & RTHAR & FCD,g & 7 & 1 & $\begin{array}{l}\text { WB, IHC, } \\
\text { MSP }\end{array}$ \\
\hline 14 & $15 / \mathrm{M}$ & $6-7$ & 7 & CPS & VPA,LEV,OXC,LTG,PHT & LTHAR & FCD,g & 6 & 2 & $\begin{array}{l}\text { WB, IHC, } \\
\text { MSP }\end{array}$ \\
\hline 15 & $23 / F$ & $7-14$ & 12 & CPS,SGS & LEV, OXC, LTG & RTHAR & $\mathrm{g}, \mathrm{nl}$ & 5 & 1 & $\begin{array}{l}\text { WB, IHC, } \\
\text { MSP }\end{array}$ \\
\hline 16 & $25 / F$ & 7 & 12 & CPS & VPA, LEV, LTG, TPM & LTHAR & $\mathrm{FCD}, \mathrm{g}$ & 4.5 & 2 & $\begin{array}{l}\text { WB, IHC, } \\
\text { MSP }\end{array}$ \\
\hline 17 & $17 / F$ & $2-4$ & 11 & CPS,SGS & VPA, CBZ, LTG & RTHAR & $\mathrm{g}$ & 6 & 2 & $\begin{array}{l}\text { WB, IHC, } \\
\text { MSP }\end{array}$ \\
\hline 18 & $10 / \mathrm{M}$ & $3-4$ & 5 & CPS & VPA, OXC, LEV, TPM & RTHAR & $\mathrm{g}, \mathrm{nl}$ & 8 & 1 & $\begin{array}{l}\text { WB, IHC, } \\
\text { MSP }\end{array}$ \\
\hline 19 & $11 / \mathrm{M}$ & 3 & 11 & CPS,SGS & VPA, OXC, LEV & RTHAR & $\mathrm{FCD}, \mathrm{g}$ & 4 & 2 & $\begin{array}{l}\text { WB, IHC, } \\
\text { MSP }\end{array}$ \\
\hline 20 & $33 / F$ & $1-2$ & 11 & SGS & OXC, LEV, TPM & LTHAR & FCD,g & 5 & 3 & $\begin{array}{l}\text { WB, IHC, } \\
\text { MSP }\end{array}$ \\
\hline 21 & $35 / F$ & $2-3$ & 30 & CPS,SGS & VPA, CBZ, CZP, PHT & LTHAR & $\mathrm{g}, \mathrm{nl}$ & 6.5 & 2 & $\begin{array}{l}\text { WB, IHC, } \\
\text { MSP }\end{array}$ \\
\hline 22 & $20 / \mathrm{M}$ & $4-6$ & 9 & CPS,SGS & VPA, LEV, OXC & RTHAR & FCD,g & 7 & 1 & $\begin{array}{l}\text { WB, IHC, } \\
\text { MSP }\end{array}$ \\
\hline 23 & $19 / \mathrm{M}$ & 7 & 15 & CPS & VPA, CBZ, TPM, LTG & LTHAR & $\mathrm{g}$ & 3 & 2 & $\begin{array}{l}\text { WB, IHC, } \\
\text { MSP }\end{array}$ \\
\hline 24 & $26 / F$ & $7-14$ & 17 & CPS & VPA, LEV, OXC, LTG & LTHAR & $\mathrm{g}, \mathrm{nl}$ & 5 & 2 & $\begin{array}{l}\text { WB, IHC, } \\
\text { MSP }\end{array}$ \\
\hline
\end{tabular}




\begin{tabular}{|c|c|c|c|c|c|c|c|c|c|c|}
\hline 25 & $27 / M$ & 7 & 22 & CPS,SGS & VPA, LEV, LTG, TPM & RTHAR & $\mathrm{FCD}, \mathrm{g}$ & 6 & 1 & $\begin{array}{l}\text { WB, IHC, } \\
\text { MSP }\end{array}$ \\
\hline 26 & $32 / F$ & $3-4$ & 13 & CPS,SGS & VPA, LTG, LEV, OXC & RTHAR & $\mathrm{FCD}, \mathrm{g}$ & 8 & 3 & $\begin{array}{l}\text { WB, IHC, } \\
\text { MSP }\end{array}$ \\
\hline 27 & $26 / M$ & 4 & 19 & CPS & VPA, CBZ, CZP, PHT & RTHAR & g,nl & 4.5 & 2 & $\begin{array}{l}\text { WB, IHC, } \\
\text { MSP }\end{array}$ \\
\hline 28 & $23 / F$ & $1-3$ & 22 & SGS & LTG, OXC, LEV & LTHAR & g & 5.5 & 1 & $\begin{array}{l}\text { WB, IHC, } \\
\text { MSP }\end{array}$ \\
\hline 29 & $27 / M$ & 7 & 16 & CPS,SGS & VPA, LEV, LTG & RTHAR & g,nl & 4 & 1 & $\begin{array}{l}\text { WB, IHC, } \\
\text { MSP }\end{array}$ \\
\hline 30 & $16 / F$ & $2-3$ & 10 & CPS & VPA,LTG,OXC,LEV,CZP & LTHAR & g & 5 & 2 & $\begin{array}{l}\text { WB, IHC, } \\
\text { MSP }\end{array}$ \\
\hline 31 & $28 / F$ & 7-14 & 14 & CPS,SGS & LTG, VPA, OXC, LEV & RTHAR & $\mathrm{FCD}, \mathrm{g}$ & 4 & 1 & $\begin{array}{l}\text { WB, IHC, } \\
\text { MSP }\end{array}$ \\
\hline 32 & $26 / M$ & $3-4$ & 23 & CPS & VPA, OXC, CZP, LEV & LTHAR & $\mathrm{FCD}, \mathrm{g}$ & 6.5 & 2 & $\begin{array}{l}\text { WB, IHC, } \\
\text { MSP }\end{array}$ \\
\hline 33 & $35 / M$ & $2-3$ & 31 & CPS & VPA, CBZ, CZP, LEV & RTHAR & g & 3 & 3 & $\begin{array}{l}\text { WB, IHC, } \\
\text { MSP }\end{array}$ \\
\hline 34 & $31 / F$ & 7 & 10 & CPS,SGS & VPA,OXC,LTG,CZP,LEV & RTHAR & FCD,g & 4.5 & 2 & $\begin{array}{l}\text { WB, IHC, } \\
\text { MSP }\end{array}$ \\
\hline
\end{tabular}

Table 2

Clinical data from 12 control subjects

\begin{tabular}{|c|c|c|c|c|c|c|c|c|}
\hline No. & Gender & Age (y) & Cause of death & Etiology diagnosis & Resection tissue & $\begin{array}{l}\text { Histological } \\
\text { examination }\end{array}$ & Seizure & Application \\
\hline 1 & $M$ & 15 & Car accident & $\mathrm{N} / \mathrm{A}$ & $\mathrm{LH}$ & $\mathrm{n}$ & None & GXP, WB, IHC, MSP \\
\hline 2 & $\mathrm{~F}$ & 26 & Car accident & $\mathrm{N} / \mathrm{A}$ & $\mathrm{LH}$ & $\mathrm{n}$ & None & GXP, WB, IHC, MSP \\
\hline 3 & $M$ & 18 & Drowning & $\mathrm{N} / \mathrm{A}$ & $\mathrm{LH}$ & $\mathrm{n}$ & None & GXP, WB, IHC, MSP \\
\hline 4 & $M$ & 24 & Car accident & $\mathrm{N} / \mathrm{A}$ & $\mathrm{LH}$ & $\mathrm{n}$ & None & WB, IHC, MSP \\
\hline 5 & M & 37 & $\mathrm{~N} / \mathrm{A}$ & Severe TBI & $\mathrm{RH}$ & $\mathrm{n}$ & None & WB, IHC, MSP \\
\hline 6 & $\mathrm{~F}$ & 46 & $\mathrm{~N} / \mathrm{A}$ & Severe TBI & $\mathrm{RH}$ & $\mathrm{n}$ & None & WB, IHC, MSP \\
\hline 7 & M & 35 & $\mathrm{~N} / \mathrm{A}$ & Severe TBI & $\mathrm{LH}$ & $\mathrm{n}$ & None & $\mathrm{IHC}$ \\
\hline 8 & M & 45 & N/A & RTG & $\mathrm{RH}$ & $\mathrm{n}$ & None & $\mathrm{IHC}$ \\
\hline 9 & $M$ & 53 & $\mathrm{~N} / \mathrm{A}$ & LTG & $\mathrm{LH}$ & $\mathrm{n}$ & None & $\mathrm{IHC}$ \\
\hline 10 & $\mathrm{~F}$ & 44 & N/A & LTG & $\mathrm{LH}$ & $\mathrm{n}$ & None & $\mathrm{IHC}$ \\
\hline 11 & $\mathrm{~F}$ & 38 & $\mathrm{~N} / \mathrm{A}$ & RTG & $\mathrm{RH}$ & $\mathrm{n}$ & None & $\mathrm{IHC}$ \\
\hline 12 & $\mathrm{~F}$ & 49 & $\mathrm{~N} / \mathrm{A}$ & LTG & $\mathrm{LH}$ & $\mathrm{n}$ & None & $\mathrm{IHC}$ \\
\hline
\end{tabular}

\section{Animals and Treatments}


Adult male SD rats (200-240 g body weight) were obtained from the Experimental Animal Center of the Fourth Military Medical University. Preparation of the lithium-pilocarpine model of temporal lobe epilepsy and how animals were grouped were described in detail in our previously published articles ${ }^{17,18}$

\section{Human mRNA profiling}

Blocks of hippocampus chosen randomly from the epileptic $(n=8)$ and control $(n=3)$ tissue groups were cut into small pieces and homogenized in $1.5 \mathrm{ml}$ Trizol (Sigma). All samples underwent the same tissue dissection and RNA extraction sample preparation protocol performed by the same individuals. Spectrophotometric evaluation of RNA quality and quantity using a microvolumetric Nanodrop ND-2000 spectrophotometer (Thermo Fisher Scientific, USA). RNA integrity was verified by gel electrophoresis. The TruSeq ${ }^{\circledR}$ RNA LT Sample Prep Kit v2 (Illumina Inc., USA) was used for high-throughput strand-specific mRNA library preparation, and the qualified libraries were sequenced by Genergy Biotechnology Co. Ltd. (Shanghai, China) using Illumina HiSeq 2500 following the manufacturer's instructions (Illumina) ${ }^{20}$. All sequencing and data analysis were conducted by Genergy Inc (Shanghai, China). A detailed description of the sequencing process is provided in the supplemental material.

\section{Surgery and intracranial EEG recording}

For EEG surgery, adult male SD rats were anaesthetized with sodium pentobarbital (50 mg/kg, i.p.). Stainless steel screws were used as electrodes, placed on top of the dura through small holes drilled through the skull and then fixed to the skull using dental cement. The locations of the electrodes were F3/4 (+2.0 mm from bregma, $2.25 \mathrm{~mm}$ lateral from midline), C3/4 (-3.0 mm from bregma, $2.75 \mathrm{~mm}$ lateral from midline), P3/4 (-7.0 $\mathrm{mm}$ from bregma, $2.75 \mathrm{~mm}$ lateral from midline) (Fig. 4B). The ground electrode was placed in the midline approximately $1.5 \mathrm{~mm}$ posterior of Lambda. EEG electrodes were wired to a head mount that was also fixed to the skull using dental cement (Fig. 4C). After 5-7 days of recovery from the surgery, EEG signals and synchronized video were recorded using the EEG-9200K (Nihon Kohden, Tokyo, Japan). The voltage differential between the pair of electrodes from each brain hemisphere were amplified with a high pass filter $(1 \mathrm{~Hz})$ and recorded. EEG signals were sampled at $400 \mathrm{~Hz}$ and videos were recorded at $30 \mathrm{frames} / \mathrm{sec}$. The detailed process is described in our previously published articles 21,22

\section{Li-Pilo-induced SE and Behavioral grading of seizures}

After $5 \mathrm{~min}$ of EEG and video recording of baseline activity, rats were first administered a single dose of methylscopolamine (1 mg/kg, i.p.) to allay the peripheral effects of pilocarpine. A single dose of pilocarpine ( $280 \mathrm{mg} / \mathrm{kg}$, i.p.) was administered approximately $25 \mathrm{~min}$ after methylscopolamine to induce acute seizures. After $15 \mathrm{~min}$, rats in the treatment group were injected with Fasudil (25 mg/kg, i.p., APExBlo Technology, USA) and returned to their home cages to observe the behavioral grade of seizures. The scoring was based on the Racine scale, as described previously (Racine, 1972) with the following stages: 0, no abnormality; 1, Mouth and facial movements; (2) Head nodding; (3) Forelimb clonus; (4) Rearing; (5) Rearing and falling. A full motor seizure, with temporary loss of postural control, is referred to as a Stage 5 motor seizure.

\section{Cortical Primary Astrocyte Cultures from adult SD Rats}

Adult male SD rats from the Experimental Animal Center of the Fourth Military Medical University were sacrificed in a CO2 chamber according to current Fourth Military Medical University (Animal Use for Research and Education Committee) guidelines. Cerebral cortex was dissected aseptically and the meninges removed. Cortices were dissociated into a cell suspension using mechanical digestion. Cells were plated in 6 well plates pre-coated with poly-L-lysine at a concentration of $5 \times 10^{5}$ cells/cm ${ }^{2}$ in modified DMEM/F12 medium [10\% FBS, 15 mM HEPES, 14.3 mM $\mathrm{NaHCO}, 1 \%$ fungizone and $0.04 \%$ gentamicin]. The first change ofmedium was performed after $24 \mathrm{~h}$ of culture. During the first week, the medium change occurred once every 2 days and, from the second week, once every 4 days. From the third week onwards, the cells received medium supplemented with $20 \% \mathrm{FBS}^{23}, 24$. Around the third to fourth weeks, cells reached confluence and were used for the experiments. The purity of the primary astrocyte cultures was assessed by immunocytochemistry for GFAP.

\section{Plasmid, shRNA and virus}

The ROCK2 shRNA sequence used to increase ROCK2 expression was 5'- ACATGCCAGGTGGAGACCTTGTTAATTGCTCTTTTTG-3' (Shanghai GenePharma). Scramble was used as negative control. ROCK2-overexpressing lentivirus were packaged in Hanbio Biotechnology (China).

\section{Double immunofluorescence}


Immunofluorescence was performed as described in our previously published articles ${ }^{25,26}$. The brain sections were incubated in a primary antibody solution containing rabbit anti-ROCK2 antibody (1:700; \#PA5-78290, Invitrogen) and mouse anti- NeuN antibody (1:1000; \#ab104224, Abcam) or mouse anti-GFAP antibody (1:1000; \#ab10062, Abcam) that was dissolved in the rinse buffer for $12 \mathrm{~h}$ at $4{ }^{\circ} \mathrm{C}$. Before secondary antibody incubation, the sections were washed 3 times for 30 min in PBS. The sections were then incubated in a secondary antibody solution containing FITC-conjugated goat anti-rabbit IgG (1:2000; \#ab6717, Abcam) and Texas Red-conjugated goat anti-mouse lgG (1:2000; \#ab6787, Abcam) in the rinse buffer for $4 \mathrm{~h}$ at $4{ }^{\circ} \mathrm{C}$. After washing 3 times for $30 \mathrm{~min}$ in PBS, the sections were coverslipped with anti-fading medium. Images of double immunofluorescence staining were obtained using a confocal laser-scanning microscope system (Nikon $\mathrm{C} 2+$, Tokyo, Japan). Images were analyzed with ImageJ software (ver. 1.46; NIH, USA).The ratios of cell number of single-positive cells and double-positive cells (\%) was evaluated as described in the article 27.

\section{Western Blot Analysis}

Total proteins from tissues or cell extracts $(50 \mu \mathrm{g} / \mathrm{gel}$ lane) were separated by SDS-PAGE, blotted, and probed with anti-ROCK2 (1:800; \#PA5-78290, Invitrogen), anti-Stat3 (1:3000; \#ab68153, Abcam), anti-p-Stat3 (1:2000; \#ab76315, Abcam), anti- $\beta$-actin (1:5000; \#ab8227, Abcam), and antiTubulin (1:5000; \#ab6160, Abcam) alone or in combination. Secondary antibodies conjugated with HRP (1:3000, Abcam, UK) were detected using the ChemiDoc XRS+ system (Bio-Rad, Hercules, USA). Bands were quantified by densitometry using ImageJ software (ver. 1.46; NIH, USA).

\section{qPCR}

Extract the RNA and generate complementary DNA through GoScript Reverse Transcription System (Promega). The qPCR assay was performed as described previously ${ }^{28}$. Primers used for qPCR analysis of ROCK2, c-Myc, Cyclin D1, GFAP, P21, P15, and $\beta$-actin are listed on Table 3.

Table 3

Oligonucleotide primers for real-time RT-PCR

\begin{tabular}{|ll|}
\hline mRNA target & Sense/anti-sense \\
\hline c-Myc & TGCCCGATCATCCCCTAGAA \\
& AGGCAGTTAGCTTGGTTTGT \\
Cyclin D1 & GAGGTGGAAAACCCGACAGT \\
& GAAATAGGGCTGCACCGAGT \\
GFAP & TAGGGCTGGTAGCATGAGGT \\
& AGATCCCGGTGGTACGAGAA \\
\hline P21 & TTGACCTGCGACCTTGAGTC \\
& GAGTGCCTCCTGGTAACTCG \\
\hline P15 & TGTGATATGTACCAGCCACAGG \\
& CGAACAGACGACGGCATACT \\
\hline -actin & GACTCACTCGCGGCTCC \\
& GGCTCCCGTTAGACACTCTC \\
\hline
\end{tabular}

\section{Flow Cytometric Analysis of the Cell Cycle}

Cell cycle assay was performed as described previously ${ }^{29}$.

\section{ChIP}

The ChIP analysis was performed using the ChIP Assay kit (Upstate Biotechnology, Charlottesville, VA). $10^{7}$ cells were crosslinked with $1 \%$ formaldehyde for $10 \mathrm{~min}$ at $37^{\circ} \mathrm{C}$ and then washed, lysed, and sonicated to generate $200-500$ bp chromatin fragments. The samples were 
precleared with $60 \mu \mathrm{l}$ of salmon sperm DNA-protein A-agarose and subsequently incubated at $4{ }^{\circ} \mathrm{C}$ overnight with $2 \mu \mathrm{Sg}$ Stat 3 antibody and rabbit IgG as control. Immunocomplexes were recovered, washed thoroughly, and eluted with the ChIP elution buffer. Following the reversal of crosslinks at $65^{\circ} \mathrm{C}$ for 4 hours, samples were extracted with phenol/chloroform, precipitated with ethanol, and then used as templates for PCR amplification. The sequences of PCR primers detecting c-Myc and Cyclin D1 promoter are available on request.

\section{Methylation Assay}

The methylation assay were performed as previously described ${ }^{29}$. Primers for the methylated promoter region were $5^{\prime}$ -

GATATACGATAGGAATTACGGGG-3' (sense) and 5'-TCTCTCTACCTTATCTAACCCG-3' (antisense), and for the unmethylated region 5'-

GATATATGATAGGAATTATGGGG-3' (sense) and 5'-CTCTACCTTATCTAACCCATTCCC-3' (antisense). The intensity of the methylated and the loading control products were analyzed by Image J software (NIH, Bethesda, MD) and compared with the base pairs of the amplicons and the cycles of individual PCR reactions. When the relative ratio of the methylated to the total DNA was more than $50 \%$, methylation was considered high, whereas it was considered low when the ratio was less than $50 \%$. Samples with no obvious methylation bands were considered as having no methylation.

\section{IHC}

Epileptic and control hippocampus tissues were collected at the tangdu Hospital of Fourth Military Medical University. The immunohistochemistry staining assay was performed and scored as previously described ${ }^{17}$. The primary antibodies for anti-ROCK2 (1:200, \#PA5-78290, Invitrogen) and anti-p-Stat3 (1:150; \#ab76315, Abcam) were applied. The protein levels were statistically analyzed by Student's t-test. Linear regression and Pearson's correlation significance were used to analyze ROCK2 and p-Stat3 correlation.

\section{Statistical analysis}

The data are expressed as the mean \pm SEM. Statistical analyses were performed in GraphPad Prism 8.0 (GraphPad Software Inc, San Diego, CA). One-way ANOVA analysis followed by Dunnett's multiple comparison test was used to determine the differences among multiple groups. Correlations between the protein levels of ROCK2 and p-Stat3 were assessed using Pearson's rank correlation test. Statistical significance was based on a value of $P<0.05$.

\section{Results}

\section{mRNA Profiling of human refractory epilepsy samples}

To gain mechanistic insight into system-level differences between normal and epileptic hippocampus tissues, we determined the gene expression profiles in 8 drug-resistant epilepsy samples and 3 control samples (Tables 1 and 2). Compared with the control samples, 1389 genes were downregulated and 1014 genes were up-regulated in refractory epilepsy samples (Fig. 1A, 1B). Further, we analyzed the top20 upregulated genes in epilepsy samples. Intriguingly, ROCK2 were found most dramatically highly expressed in epilepsy samples (Fig. 1C), suggesting the high levels of ROCK2 might be associated with epilepsy generation.

\section{Elevated expression of ROCK2 in human epileptic hippocampus by the hypomethylation of ROCK2 promoter}

To evaluate the role of ROCK2 in epilepsy, we firstly analyzed the expression levels of ROCK2 in the normal ( $n=6)$ and epileptic ( $n=34)$ hippocampus tissues (Tables 1 and 2). Intriguingly, the densitometric analysis of western blots shows that ROCK2 was highly expressed in the hippocampus tissue of drug-resistant TLE patients (Fig.2B). The ROCK2 product OD in patients was significantly higher than that in controls (Fig. $2 \mathrm{C}$ ). To determine the underlying mechanisms, we examined the potential changes in ROCK2 gene promoter region. Samples from TLE patients and control tissue samples were collected for methylation analysis by nested PCR. Methylation-specific PCR (MSP) analysis shows that ROCK2 promoter region was highly methylated in control samples and only 1 of 6 TLE patients (Fig.2D). Further, a large scale of MSP analysis identified the hypermethylation of ROCK2 promoter in 9 of 12 normal tissues and 6 of 34 TLE patients (Fig.2E). Thus, epileptogenesis appears to be negatively associated with the hypomethylation of ROCK2 promoter and concomitant induced its expression.

\section{Increased expression of ROCK2 in hippocampal and neocortical epilepsy}


Rats were divided randomly into eight subgroups of ten rats each and injected with lithium and $24 \mathrm{~h}$ later with saline (one subgroup) or the convulsant pilocarpine (seven subgroups) to establish a control and spontaneous epilepsy model groups. Neural ROCK2 expression was then determined at different times after seizure induction $(1,3,5,7,15,30$, or 60 days). The lithium-pilocarpine model of epilepsy in rats were prepared and evaluated as previously described ${ }^{17}$. Western blot analysis of brain lysates prepared at these different times after pilocarpine treatment revealed increased ROCK2 expression compared with controls (Fig. 3). Compared with the control group, expression of ROCK2 in the rat temporal lobe increased gradually during the acute period (day 1-3) (Fig. 3A) before increasing obviously during the latent period (days 5-15) and the chronic phase (days 30-60). Expression of ROCK2 as expressed by product OD relative to that of $\beta$-actin was significantly different between control and every epilepsy subgroup ( $p<0.05$ by Tukey's HSD test) except for the 1-day subgroup ( $p>0.05)$ (Fig. 3B). The expression of ROCK2 in epileptic hippocampus (Fig. 3C, D) followed a similar trend to that in the temporal cortex, with significant pair-wise differences in ROCK2 expression between the control and every epilepsy subgroup $(p<0.05)$ except the 1-day subgroup. The expression of ROCK2 in the hippocampus increased even more obviously at $15 \llbracket 30$ and 60 days after seizures( $p<0.01)$ (Fig. 3D).

\section{Fasudil alleviates rat epileptic seizures in vivo and protects against Li-Pilo-induced SE}

Li-Pilo-induced epileptic rat model is one of the most widely used on epileptogenesis research and has been assess the seizures based on changes in the Racine stages. The rats were then divided into 3 groups (saline group $\square$ PILO group and Fasudil treatment group). Fasudil, a specific Rhokinase inhibitor, was used to treat the rats following Li-Pilo induced seizures for 2 weeks. Then seizures were induced by the low dose PILO (200 $\mathrm{mg} / \mathrm{kg}$, i.p.) again and assessed the effects of Fasudil on the epileptogenesis. In the present study, the video-EEG recording and racine stages were used to analyze seizure severity between Fasudil treatment group and PILO group. After administration of PILO, stage 3 behavioral scores earlier appeared in PILO group compared with Fasudil treatment group, stage 4 and 5 behavioral scores did not appear in Fasudil treated rats (Fig. 4A). Further, we examined hippocampal EEG activity in LiCl-Pilo epileptic rats at symptomatic stages (Fig. 4D). The latency (min) of generalized tonicclonic seizures (GTCS) in the PILO group was 28.39 \pm 2.10 , while the latency in the Fasudil treatment group was $53.01 \pm 4.16$ (Fig. $4 \mathrm{E}$ ). The EEG showed $14.96 \pm 0.43$ spikes per second in the PILO group and $6.88 \pm 0.13$ spikes per second in the Fasudil treatment group (Fig. 4F). The spike amplitude $(\mu \mathrm{V})$ of EEG in PILO group and Fasudil treatment group were 232.83 \pm 13.61 and 108.72 \pm 2.94 , respectively (Fig. 4G).

\section{ROCK2 activates Stat3 pathway in Pilocarpine-Treated Epileptic Rats}

To further investigate the role of ROCK2 in regulation of epileptogenesis, we determined the downstream targets in SE rat model. JAK/Stat3 pathway was previously shown constitutively activated during brain injuries like SE, and plays a key role in epilepsy development ${ }^{30,31}$. In the present study, we observed the phosphorylation and activation of Stat3 following SE. Intriguingly, Fassudil administration significantly decreased Stat3 phosphorylation, indicating ROCK2 potentially contributes to epileptogenesis through the regulation of Stat3 pathway (Fig. 5A, 4B). Further, we determined the downstream target gene expressions of Stat3. Myc and Cyclin D1, which play important roles in regulating cell proliferation and cell cycle progression, were induced following SE and significantly decreased after Fassudil treatment (Fig. 5C). Therefore, we suppose that ROCK2 induces cell proliferation via the activation of Stat3 pathway in brain tissue, and further contributes to the development and progression of epilepsy.

\section{ROCK2 is predominantly expressed in astrocytes during epileptogenesis}

Above data showed the elevated expression of ROCK2 in the brain tissues of TLE patients. To further address ROCK2 function in epileptogenesis, we detected the localization of ROCK2 in both neuron and astrocytes of rat brain tissue. The co-localization of ROCK2 with NeuN or GFAP was observed in normal brain tissue, indicating ROCK2 expression in certain level is necessary for the biological function of both neuron and astrocytes (Fig. 6A, 6C). Whereas, from the third day after SE, ROCK2 expression in astrocytes was significantly increased in contrast with the constant level of ROCK2 in neuron tissue. Quantitative analysis showed nearly 2-fold increase of ROCK2 expression in astrocytes (Fig. 6B, 6D). Thus, we show that ROCK2 is predominantly expressed in astrocytes during epileptogenesis, indicating ROCK2 possibly induces epilepsy progression through the specific regulation of astrocyte function.

\section{ROCK2 induces epileptogenesis by activating astrocyte cell cycle progression via Stat3 pathway}

Since accumulated evidence showed that astrogliosis plays important roles in epileptogenesis, it is reasonable to consider the involvement of ROCK2 in astrocyte proliferation and activation ${ }^{32,33}$. Based on this hypothesis, we set up the primary astrocytes from rat brain tissue, whose phenotype has been confirmed with GFAP in our previous study ${ }^{17}$. To investigate how ROCK2 confers astrocyte behavior, we overexpressed ROCK2 in astrocytes. Expectedly, the phosphorylation of Stat3 was dramatically induced by ROCK2 expression (Fig. 7A). Further, GFAP, the marker

Page $8 / 26$ 
of astrocyte proliferation potential and Stat3 target gene, was induced by ROCK2 (Fig. 7B). During cell cycle analysis, we found ROCK2 accelerated astrocyte G1-S transition, indicating ROCK2 induces astrocyte proliferation which confers gliosis potentially through the regulation of cell cycle progression (Fig. 7C). Accordingly, Fasudil treatment blocked ROCK2 induction of pStat3 and GFAP expressions, and G1-S transition, which further confirmed our hypothesis (Fig. 7A-C).

To fully address the related mechanism, we then determined whether ROCK2 regulates cell cycle transition via Stat3 pathway. Previous data revealed Stat3 target genes, Myc and Cyclin D1, were ROCK2 dependently induced in SE rat brain tissue (Fig. 5C). Further study showed that Myc and Cyclin D1 were remarkably induced by ROCK2 in astrocytes (Fig. 7D). The CDK inhibitor p21 and p15, which are Myc direct targets, were decreased after ROCK2 overexpression. Additionally, ChIP experiments indicated that the binding of Stat 3 to Myc and Cyclin D1 promoter was dramatically increased by ROCK2 overexpression (Fig. 7E). In the presence of Fasudil, ROCK2-induced expressions and Stat3 occupancies of Myc and cyclin D1 were significantly abolished in astrocytes (Fig. 7D, 7E). Taken together, these data suggest that ROCK2 induces the development of epilepsy, potentially through the activation of astrocyte cell cycle progression via Stat3 pathway.

\section{ROCK2 is associated with Stat3 activation in TLE patients}

To evaluate the clinical significance of ROCK2 induction of Stat3 pathway during epilepsy, we performed immunohistochemical analysis to assess the expressions and association of ROCK2 and pStat3 in TLE patients. The results revealed that both ROCK2 and pStat3 expressions were increased in patient samples $(n=34)$ compared with control samples $(n=12)(F i g .8 A)$. Indeed, in patient tissues versus control, ROCK2 levels were 7.82 versus 3.92 (Fig. 8B), respectively, and pStat3 levels were 6.88 versus 2.67 (Fig. 8C), respectively. An association study showed that ROCK2 expression positively correlated with pStat3 expression in the entire sample population (Fig. 8D) ( $p<0.001$, ANOVA). Thus, the epilepsy-associated increase of ROCK2 is associated with the increased surface activation of Stat3 pathway.

\section{Discussion}

Active RhoA binds to its downstream targets to initiate numerous cellular responses. ROCK, a serine/threonine kinase, is one of the most studied RhoA effectors and is involved in regulation of the actin cytoskeleton, cell proliferation, focal adhesion /stress fiber formation, and smooth muscle contraction $^{34}$. The current study explored the role of ROCK2 in epilepsy, focusing on the possibility that ROCK2 inhibition is required for the suppression of seizures. Although ROCK2 has not previously been suspected to play a role in epilepsy, our data demonstrate the importance of ROCK2 in the Li-Pilo model and clinical epilepsy sample, by the inhibition of ROCK2 associated reduction in seizure activity. Thus, we identify ROCK2 as a key regulator of epilepsy, and a potentially novel therapeutic target for the prevention and treatment of epilepsy.

In this study, ROCK2 expression was firstly evaluated in normal and epileptic brain tissue. We show that increased ROCK2 expression in human epileptic brain tissue. The underlying mechanism study suggests that ROCK2 gene promoter region was hypomethylated in TLE patients, which potentially contributes to the elevated expression of ROCK2. To our knowledge this is the first study providing direct evidence of increased ROCK2 expression in patients with drug-resistant TLE. Further, the highly isomorphic with human cases of TLE animal model was used, namely the model of TLE with pilocarpine-induced seizures ${ }^{35}$. In order to analyze the dynamics of epileptic seizure-induced alterations on the brain, samples taken from seizure experiencing animals $1,3,5,7,15,30$, and 60 days after proconvulsive agent administration were examined. Our study shows that the expression of ROCK2 protein increased dramatically in epilepsy rat cortex and hippocampus. Thus, we set up the association of ROCK2 with epileptogenesis.

Astrocytes are now known to be intimately involved in the pathogenesis of many neurological disorders, including epilepsy ${ }^{32,36}$. The reactive astrocytosis may facilitate the development of epileptic seizures ${ }^{37}$. The cells of the high ROCK2 expression were found in pyramidal neurons of the hippocampus and cerebral cortex and Purkinje cells of the cerebellum ${ }^{10}$. By immuno-fluorescence, ROCK2 was mainly localized in the neurons in our study. A previous study has shown that RhoA activation in the cortex and hippocampus $24 \mathrm{~h}$ after traumatic brain injury-related seizures ${ }^{4}$. In this study, we observed the expression of ROCK2 in hippocampal astrocytes in the Li-Pilo model. GFAP is the main intermediate filament protein in mature astrocytes. It has been demonstrated that GFAP-immunoreactive reactive astrocytes exhibited markedly increased RhoA expression in response to spinal cord injuries after kainic acid treatment ${ }^{38}$. We show reactive astrocyte co-localization with ROCK2 expression in the Li-Pilotreated hippocampus, which is consistent with the Rho-ROCK pathway induces the generation of a reactive astrogliosis ${ }^{39}$. However, the expression of ROCK2-positive neurons is not induced by Li-Pilo treatment, indicating the increased expression of ROCK2 may be associated with pathological changes predominantly in astrocytes.

JAK/Stat3 pathway plays an important role in normal development and a number of disease processes, including epileptogenesis ${ }^{30,40,41}$. Recent evidence shows the aberrant activation of this pathway after a variety of brain insults that can lead to epilepsy, such as pilocarpine or kainateinduced $\mathrm{SE}^{30,40}$, and traumatic brain injury ${ }^{42}$. Whereas, the potential mechanism has not been fully addressed. Our study shows the highly expression ROCK2 can activate Stat3 activation in astrocytes, and further contribute to the development of epilepsy via the induction of astrogliosis. Thus, inhibiting ROCK2 expression or Stat3 phosphorylation may be a therapeutic approach to inhibit primary epileptogenesis. 
Because ROCK is a serine/threonine kinase and cannot directly mediate tyrosine kinase phosphorylation of Stat3, it is likely that other intracellular signaling components, which may interface between ROCK and STAT3, are also engaged in this ROCK2-dependent activation of Stat3 ${ }^{43}$, 44 .

The transcription factor Stat3 is known to have important roles in regulating gene expression, specifically increasing genes important to cell proliferation and cell cycle progression ${ }^{31,45}$. In this study, we determined how ROCK2 induces epilepsy via Stat3 pathway, and found Stat3 target genes, Myc and Cyclin D1, were significantly induced by ROCK2. Further, ROCK2 increased the occupancies of Stat3 on Myc and Cyclin D1 promoters, and accelerated astrocyte cell cycle progression. The CDK inhibitor protein p21 and p15 are important downstream targets of Myc and critical for cell cycle inhibition ${ }^{46,47}$. We show here that p21 and p15 were further suppressed by ROCK2 activation of Myc via Stat3 pathway. Thus, we show ROCK2 induces astrocyte cell cycle progression in regulation of Stat3 pathway, and further contribute to the development of epileptic seizures. These experiments may explain, on one hand, the requirement of Stat3 for ROCK2-mediated activation of astrogliosis and, on the other, the ability of ROCK2 to provide a second input of cell cycle regulation for epileptogenesis in addition to the regulation of actin cytoskeleton dynamics and glutamatergic synaptic function as mentioned before 48,49 .

Since the continuous activation of astrocytosis confers epileptogenesis, our study provides the evidence of ROCK2 involvement in astrogliosis, which suggests ROCK2 could be considered as a potential target for the treatment of TLE patients. Further, we aimed to elucidate the role of ROCK2 activation of Stat3 pathway in the development and progression of epilepsy, specifically whether brief inhibition of ROCK2 affects severity of SE. We thus introduced ROCK2 inhibitor Fasudil in the present study. Fasudil has been a commonly used rock signal inhibitor, which significantly reduced the duration of tonic hindlimb extensions and recovery latency for righting reflex in maximal electroconvulsive shock induced epilepsy mice, and prolonged the onset time of seizures in PTZ epilepsy mice ${ }^{15}$. Song et al. found that FH is a potential treatment option to prevent or reverse cognitive dysfunction in Li-Pilo-induced epileptic rat model ${ }^{50}$. Furthermore, Çarçak et al. have demonstrated a single systemic administration of Fasudil partially reduced the duration and frequency of absence seizure ${ }^{51}$. In the present study, we confirmed that the Racine scale score of the Fasudil treatment group was significantly lower than that of the PiLo group. Fasudil prolonged the latency of generalized seizures and reduced the amplitude and frequency of EEG. Based on the results of this article and the clinical practice of our epilepsy center (data to be published), we believe that ROCK2 inhibitors may have better therapeutic effects on GTCS and SE.

Furthermore, immunohistochemical staining of epileptic samples for ROCK2 and pStat3 also showed a correlation of protein accumulation between these two proteins, which is also positively correlated with the development of epilepsy. Thus ROCK2-mediated activation of Stat3 pathway likely serves as an important guardian for epileptogenesis. It should be noted that, as is often observed among clinical samples, no 1:1 correlation between ROCK2 and pStat3 expression in tissues was observed. Thus, in addition to ROCK2, other factors may also contribute to the activation of Stat3 pathway during epilepsy progression.

Taken together, our work provides the evidence that elevated expression of ROCK2 due to its promoter hypomethylation may contribute to the etiology of epilepsy. In terms of epileptogenesis, ROCK2 accelerates astrocytes cell cycle progression via the activation of Stat3 pathway, and further induces astrogliosis. As such, Targeting ROCK2 may lead to the inhibition of Stat3 phosphorylation in astrocytes, and that will be especially effective at restricting epilepsy progression. These data will extend the role of ROCK2 in the pathophysiology of drug-resistant TLE and define ROCK2 and its downstream signaling pathways as potential targets for therapeutic intervention for drug-resistant TLE.

\section{Abbreviations}

TLE, Temporal lobe epilepsy; Li-Pilo, Lithium-pilocarpine; CNS, Central nervous system; ROCK囚 Rho-associated coiled-coil forming protein kinase; PTZ, Pentylenetetrazole; MES, Maximal electroconvulsive shock; IHC, Immunohistochemistry; CDK, cyclin-dependent kinase; AEDs, Anti-epileptic drugs; SD, Sprague Dawley; EEG, Electroencephalography; SE, Status epilepticus; FBS, Fetal bovine serum; NeuN, Neuronal nuclei; GFAP, Glial fibrillary acidic protein; HRP, horseradish peroxidase; qPCR, Quantitative PCR; ChIP, Chromatin immunoprecipitation; FLAIR-MRI, Fluid-attenuated inversion recovery magnetic resonance imaging; FDG-PET; Fluorodeoxyglucose-positron emission tomography; GTCS, Generalized tonic-clonic seizures.

\section{Declarations}

\section{Authors' contributions}

BL and LLS designed the research and wrote the manuscript; BL and LLS performed the study supervision. LJS, HZ and JGJ conducted the experiments, analyzed the data and wrote the manuscript; CW, XPQ, XJ, LG, GL and DLW participated in scientific discussions, analyzed the data and provided detailed feedback on all areas of the project. All authors read and approved the final manuscript.

\section{Acknowledgements}


We sincerely thank the patients and their families for their participation and support in this study.

\section{Funding}

This work was supported by the National Natural Science Foundation of China (No. 81572697, 81874156 and 81971206 ).

\section{Availability of data and materials}

The data used in our study are available from the authors on reasonable request.

\section{Ethics approval and consent to participate}

All the animal experiments were approved by the Local Committees of the Use of the Laboratory Animals, Fourth Military Medical University (Xi'an, China) and were performed in accordance with the Guidelines for Animal Experimentation of the Fourth Military Medical University and the National Institute of Health Guide for the Care and Use of Laboratory Animals (NIH Publications No. 80-23, revised1996).The human experimental protocols were approved by the Committee on Human Research at the Fourth Military Medical University. All procedures were in accordance with the principles of the Helsinki Declaration. All patients received a full description of the study and gave written informed consent.

\section{Consent for publication}

Not applicable.

\section{Competing Interests}

The authors declare no potential conflict of interest.

\section{References}

1. Van Aelst L, D'Souza-Schorey C. Rho GTPases and signaling networks. Genes \& development 1997;11:2295-2322.

2. Yuan XB, Jin M, Xu X, et al. Signalling and crosstalk of Rho GTPases in mediating axon guidance. Nature cell biology 2003;5:38-45.

3. Nakayama AY, Harms MB, Luo L. Small GTPases Rac and Rho in the maintenance of dendritic spines and branches in hippocampal pyramidal neurons. The Journal of neuroscience : the official journal of the Society for Neuroscience 2000;20:5329-5338.

4. Dubreuil Cl, Marklund N, Deschamps K, Mclntosh TK, McKerracher L. Activation of Rho after traumatic brain injury and seizure in rats. Experimental neurology 2006;198:361-369.

5. Nakagawa O, Fujisawa K, Ishizaki T, Saito Y, Nakao K, Narumiya S. ROCK-I and ROCK-II, two isoforms of Rho-associated coiled-coil forming protein serine/threonine kinase in mice. FEBS letters 1996;392:189-193.

6. Ishizaki T, Maekawa M, Fujisawa K, et al. The small GTP-binding protein Rho binds to and activates a $160 \mathrm{kDa}$ Ser/Thr protein kinase homologous to myotonic dystrophy kinase. The EMBO journal 1996;15:1885-1893.

7. Leung T, Chen XQ, Manser E, Lim L. The p160 RhoA-binding kinase ROK alpha is a member of a kinase family and is involved in the reorganization of the cytoskeleton. Molecular and cellular biology 1996;16:5313-5327.

8. Wibberley A, Chen Z, Hu E, Hieble JP, Westfall TD. Expression and functional role of Rho-kinase in rat urinary bladder smooth muscle. British journal of pharmacology 2003;138:757-766.

9. Janjua N, Mayer SA. Cerebral vasospasm after subarachnoid hemorrhage. Current opinion in critical care 2003;9:113-119.

10. Hashimoto R, Nakamura Y, Kosako H, et al. Distribution of Rho-kinase in the bovine brain. Biochemical and biophysical research communications 1999;263:575-579.

11. Fournier AE, Takizawa BT, Strittmatter SM. Rho kinase inhibition enhances axonal regeneration in the injured CNS. The Journal of neuroscience : the official journal of the Society for Neuroscience 2003;23:1416-1423.

12. O'Kane EM, Stone TW, Morris BJ. Increased long-term potentiation in the CA1 region of rat hippocampus via modulation of GTPase signalling or inhibition of Rho kinase. Neuropharmacology 2004;46:879-887.

13. Dash PK, Orsi SA, Moody M, Moore AN. A role for hippocampal Rho-ROCK pathway in long-term spatial memory. Biochemical and biophysical research communications 2004;322:893-898.

14. Mueller BK, Mack H, Teusch N. Rho kinase, a promising drug target for neurological disorders. Nature reviews Drug discovery 2005;4:387-398. 
15. Inan S, Buyukafsar K. Antiepileptic effects of two Rho-kinase inhibitors, Y-27632 and fasudil, in mice. British journal of pharmacology 2008;155:44-51.

16. Jeon BT, Jeong EA, Park SY, et al. The Rho-kinase (ROCK) inhibitor Y-27632 protects against excitotoxicity-induced neuronal death in vivo and in vitro. Neurotoxicity research 2013;23:238-248.

17. Liu B, Niu L, Shen MZ, et al. Decreased astroglial monocarboxylate transporter 4 expression in temporal lobe epilepsy. Mol Neurobiol 2014;50:327-338.

18. Wu H, Wang C, Liu B, et al. Altered Expression Pattern of Acid-Sensing lon Channel Isoforms in Piriform Cortex After Seizures. Mol Neurobiol 2016;53:1782-1793.

19. Zhang H, Gao G, Zhang Y, et al. Glucose Deficiency Elevates Acid-Sensing lon Channel 2a Expression and Increases Seizure Susceptibility in Temporal Lobe Epilepsy. Sci Rep 2017;7:5870.

20. Loman NJ, Constantinidou C, Christner M, et al. A culture-independent sequence-based metagenomics approach to the investigation of an outbreak of Shiga-toxigenic Escherichia coli 0104:H4. JAMA 2013;309:1502-1510.

21. Guo H, Zhang H, Kuang Y, et al. Electrical stimulation of the substantia nigra pars reticulata (SNr) suppresses chemically induced neocortical seizures in rats. J Mol Neurosci 2014;53:546-552.

22. Yao QH, Zhang H, Wang HW, Jing XR, Guo H, Gao GD. Low- and high-frequency electric cortical stimulation suppress the ferric chlorideinduced seizures in rats. Neurosci Lett 2008;430:187-190.

23. Bellaver B, Souza DG, Souza DO, Quincozes-Santos A. Hippocampal Astrocyte Cultures from Adult and Aged Rats Reproduce Changes in Glial Functionality Observed in the Aging Brain. Mol Neurobiol 2017;54:2969-2985.

24. Longoni A, Bellaver B, Bobermin LD, et al. Homocysteine Induces Glial Reactivity in Adult Rat Astrocyte Cultures. Mol Neurobiol 2018;55:19661976.

25. Liu B, Zhang B, Wang T, et al. Increased expression of urokinase-type plasminogen activator receptor in the frontal cortex of patients with intractable frontal lobe epilepsy. J Neurosci Res 2010;88:2747-2754.

26. Liu B, Wang T, Wang L, Wang C, Zhang H, Gao GD. Up-regulation of major vault protein in the frontal cortex of patients with intractable frontal lobe epilepsy. J Neurol Sci 2011;308:88-93.

27. Nakamura Y, Kitada M, Satoh F, et al. Intratumoral heterogeneity of steroidogenesis in aldosterone-producing adenoma revealed by intensive double- and triple-immunostaining for CYP11B2/B1 and CYP17. Mol Cell Endocrinol 2016;422:57-63.

28. Meng Q, Li S, Liu Y, et al. Circular RNA circSCAF11 Accelerates the Glioma Tumorigenesis through the miR-421/SP1/VEGFA Axis. Mol Ther Nucleic Acids 2019;17:669-677.

29. Liu B, Wang L, Shen LL, et al. RNAi-mediated inhibition of presenilin 2 inhibits glioma cell growth and invasion and is involved in the regulation of Nrg1/ErbB signaling. Neuro Oncol 2012;14:994-1006.

30. Lund IV, Hu Y, Raol YH, et al. BDNF selectively regulates GABAA receptor transcription by activation of the JAK/STAT pathway. Sci Signal 2008;1:ra9.

31. Grabenstatter HL, Del Angel YC, Carlsen J, et al. The effect of STAT3 inhibition on status epilepticus and subsequent spontaneous seizures in the pilocarpine model of acquired epilepsy. Neurobiology of disease 2014;62:73-85.

32. Pollen DA, Trachtenberg MC. Neuroglia: gliosis and focal epilepsy. Science 1970;167:1252-1253.

33. Goc J, Liu JY, Sisodiya SM, Thom M. A spatiotemporal study of gliosis in relation to depth electrode tracks in drug-resistant epilepsy. The European journal of neuroscience 2014;39:2151-2162.

34. Inoue S, Saito M, Takenaka A. Hydroxyfasudil ameliorates bladder dysfunction in male spontaneously hypertensive rats. Urology 2012;79:1186 e1189-1114.

35. Curia G, Longo D, Biagini G, Jones RS, Avoli M. The pilocarpine model of temporal lobe epilepsy. Journal of neuroscience methods 2008;172:143-157.

36. Harris AB. Cortical neuroglia in experimental epilepsy. Experimental neurology 1975;49:691-715.

37. de Lanerolle NC, Lee TS, Spencer DD. Astrocytes and epilepsy. Neurotherapeutics : the journal of the American Society for Experimental NeuroTherapeutics 2010;7:424-438.

38. Erschbamer MK, Hofstetter CP, Olson L. RhoA, RhoB, RhoC, Rac1, Cdc42, and Tc10 mRNA levels in spinal cord, sensory ganglia, and corticospinal tract neurons and long-lasting specific changes following spinal cord injury. The Journal of comparative neurology 2005;484:224-233.

39. John GR, Chen L, Rivieccio MA, Melendez-Vasquez CV, Hartley A, Brosnan CF. Interleukin-1beta induces a reactive astroglial phenotype via deactivation of the Rho GTPase-Rock axis. The Journal of neuroscience : the official journal of the Society for Neuroscience 2004;24:28372845.

40. Choi JS, Kim SY, Park HJ, et al. Upregulation of gp130 and differential activation of STAT and p42/44 MAPK in the rat hippocampus following kainic acid-induced seizures. Brain Res Mol Brain Res 2003;119:10-18.

Page 12/26 
41. Xu Z, Xue T, Zhang Z, et al. Role of signal transducer and activator of transcription-3 in up-regulation of GFAP after epilepsy. Neurochem Res 2011;36:2208-2215.

42. Raible DJ, Frey LC, Cruz Del Angel Y, Russek SJ, Brooks-Kayal AR. GABA(A) receptor regulation after experimental traumatic brain injury. Journal of neurotrauma 2012;29:2548-2554.

43. Sanz-Moreno V, Gaggioli C, Yeo M, et al. ROCK and JAK1 signaling cooperate to control actomyosin contractility in tumor cells and stroma. Cancer cell 2011;20:229-245.

44. Zanin-Zhorov A, Weiss JM, Nyuydzefe MS, et al. Selective oral ROCK2 inhibitor down-regulates IL-21 and IL-17 secretion in human T cells via STAT3-dependent mechanism. Proceedings of the National Academy of Sciences of the United States of America 2014;111:16814-16819.

45. Shirogane T, Fukada T, Muller JM, Shima DT, Hibi M, Hirano T. Synergistic roles for Pim-1 and c-Myc in STAT3-mediated cell cycle progression and antiapoptosis. Immunity 1999;11:709-719.

46. Warner BJ, Blain SW, Seoane J, Massague J. Myc downregulation by transforming growth factor beta required for activation of the p15(Ink4b) G(1) arrest pathway. Molecular and cellular biology 1999;19:5913-5922.

47. Gartel AL, Ye X, Goufman E, et al. Myc represses the p21(WAF1/CIP1) promoter and interacts with Sp1/Sp3. Proceedings of the National Academy of Sciences of the United States of America 2001;98:4510-4515.

48. Saneyoshi T, Fortin DA, Soderling TR. Regulation of spine and synapse formation by activity-dependent intracellular signaling pathways. Current opinion in neurobiology 2010;20:108-115.

49. Matsuzaki M, Honkura N, Ellis-Davies GC, Kasai H. Structural basis of long-term potentiation in single dendritic spines. Nature 2004;429:761766.

50. Song X, He R, Han W, et al. Protective effects of the ROCK inhibitor fasudil against cognitive dysfunction following status epilepticus in male rats. J Neurosci Res 2019;97:506-519.

51. Carcak N, Yavuz M, Eryigit Karamahmutoglu T, et al. Suppressive effect of Rho-kinase inhibitors Y-27632 and fasudil on spike-and-wave discharges in genetic absence epilepsy rats from Strasbourg (GAERS). Naunyn Schmiedebergs Arch Pharmacol 2018;391:1275-1283.

\section{Figures}
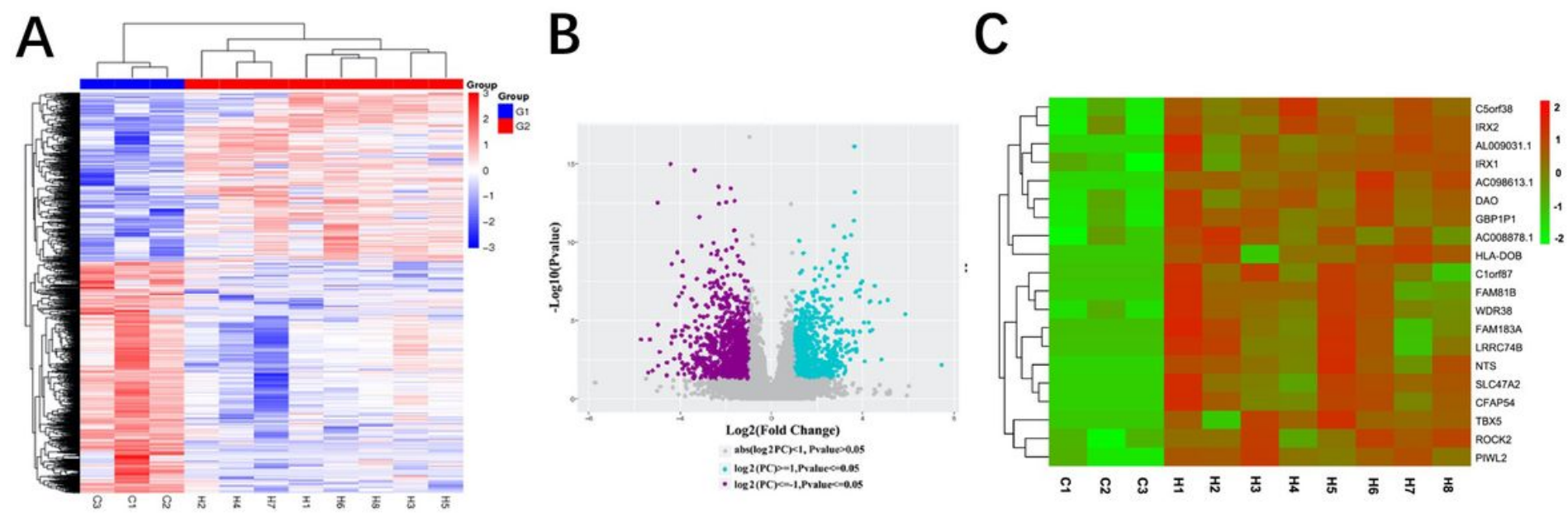

Figure 1

mRNA Profiling of human refractory epilepsy samples. (A-B) Heatmap (A) and volcano plot (B) analysis were performed to determine the genes differentially expressed in 8 drug-resistant epilepsy samples and 3 control samples. (C) The top20 upregulated genes were identified and analyzed by heatmap. 

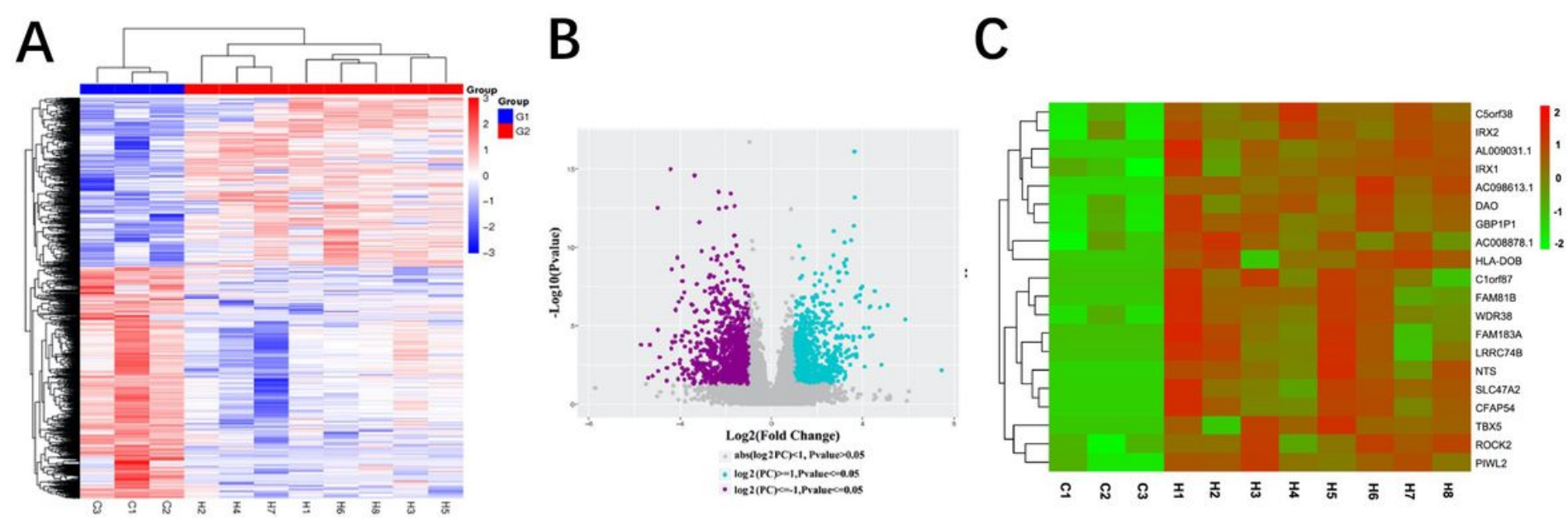

Figure 1

mRNA Profiling of human refractory epilepsy samples. (A-B) Heatmap (A) and volcano plot (B) analysis were performed to determine the genes differentially expressed in 8 drug-resistant epilepsy samples and 3 control samples. (C) The top20 upregulated genes were identified and analyzed by heatmap.

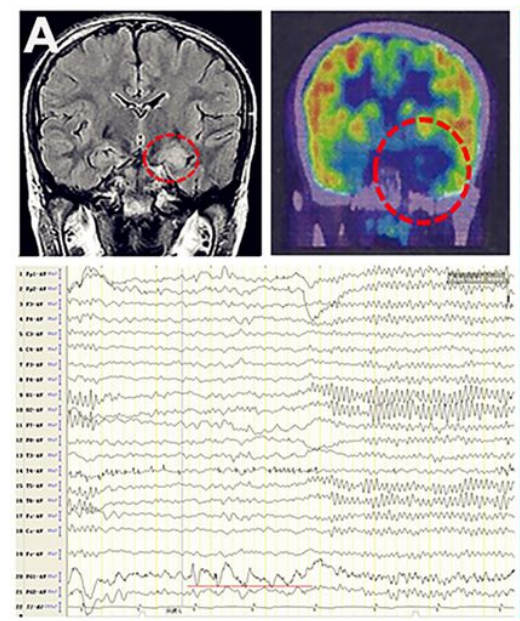

B

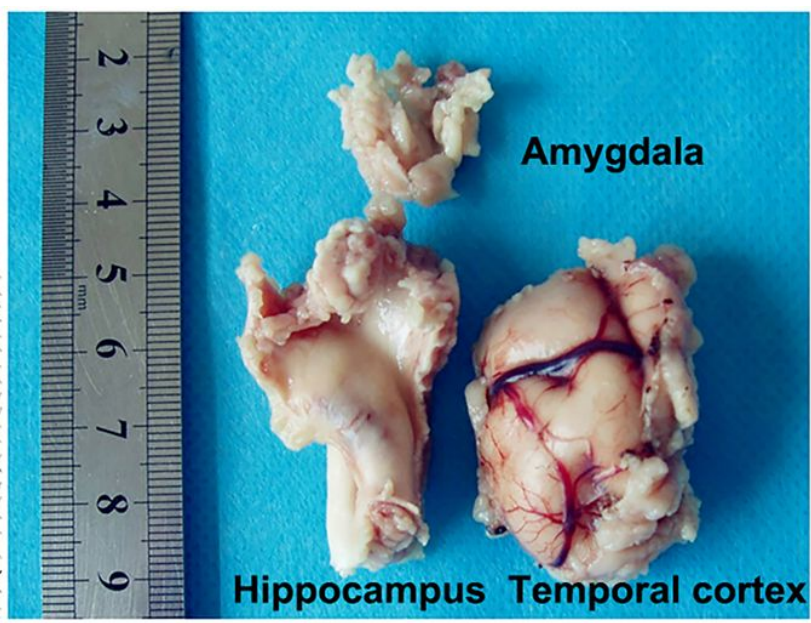

C $\begin{array}{llllllll}\text { C1 } & \text { P1 } & \text { P2 } & \text { P3 } & \text { P4 } & \text { P5 } & \text { P6 }\end{array}$

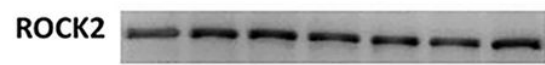

$\beta$-actin

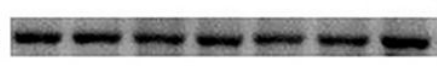

D

\section{E}
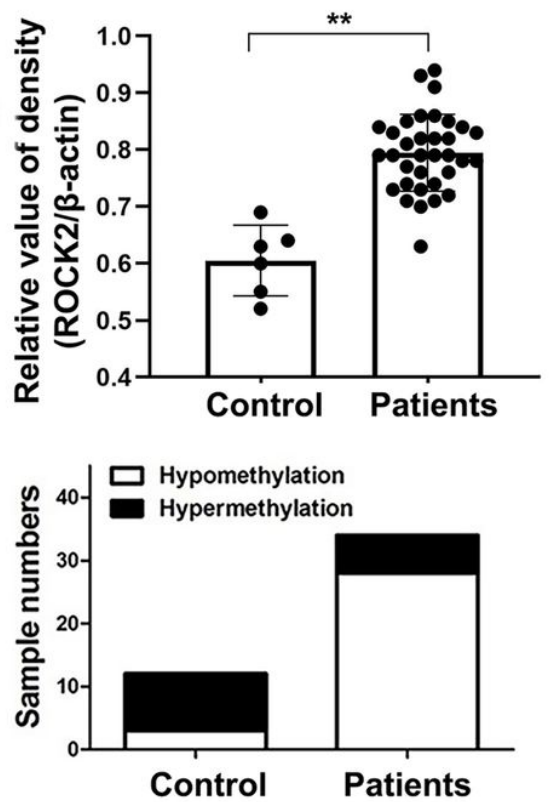

Figure 2 
Elevated hippocampal ROCK2 expression by the hypomethylation of ROCK2 promoter. (A) Patient 3's pre-operative assessment and surgical resection specimens: coronal FLAIR-MRI of the head showed abnormal high signal intensity in the left hippocampus (left), FDG-PET revealed hypometabolic lesions in the left mesial temporal lobe (middle), interictal electroencephalography showed positive sharp slow waves in the left mesial temporal region (below), and the anterior temporal lobe, hippocampus and amygdala were immediately stored in the epilepsy brain bank after surgical resection (right). (B) Representative western blot assays of ROCK2 expression in the hippocampus of controls and patients with TLE. (C) Comparison of the Western blot intensity ratios of ROCK2/ $\beta$-actin in the hippocampus of controls ( $n=6)$ and patients with TLE ( $n=34$ ). (D) Representative methylation status of the ROCK2 promoter region in the hippocampus of controls and patients with TLE. (E) Statistical graphs show the comparison of the hypermethylation of ROCK2 promoter in the hippocampus of controls $(n=12)$ and patients with TLE ( $n=34)$. The experiments were repeated 3 times. P patients with epilepsy, C control. Data were analyzed using unpaired t-tests. ${ }^{*} \mathrm{P}<0.01$.

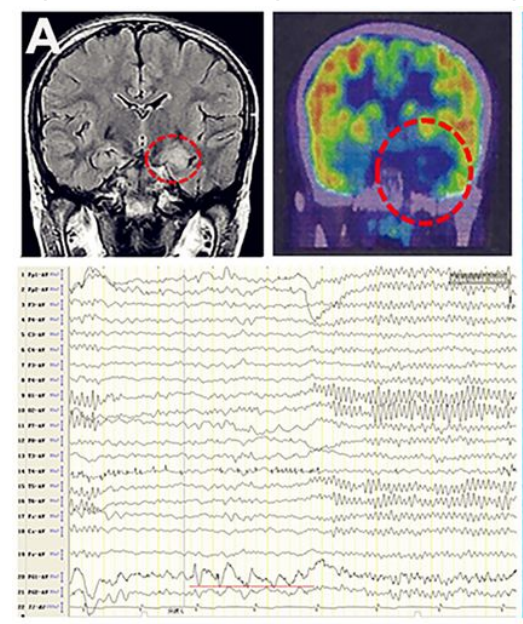

B

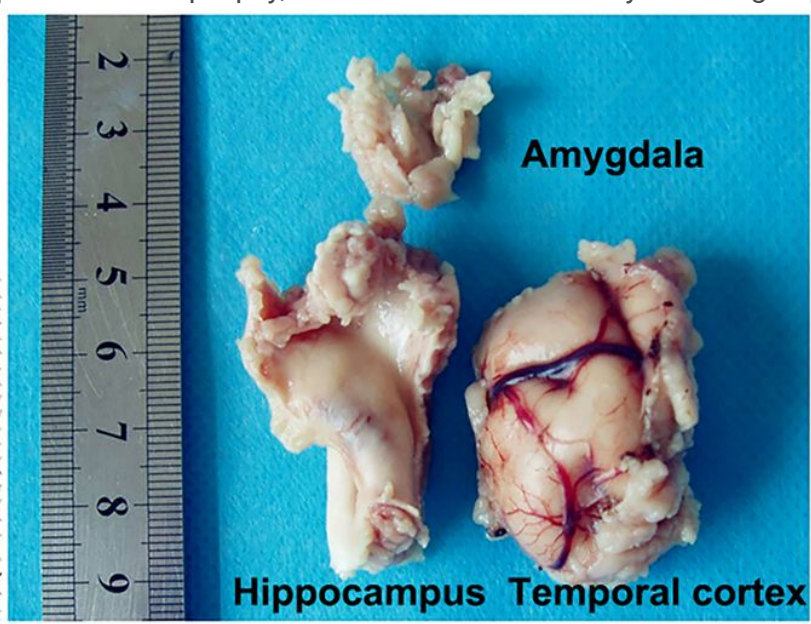

C $\begin{array}{lllllll}\text { C1 } & \text { P1 } & \text { P2 } & \text { P3 } & \text { P4 } & \text { P5 } & \text { P6 }\end{array}$ ROCK2

$\beta$-actin
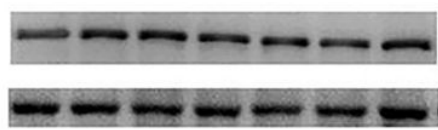

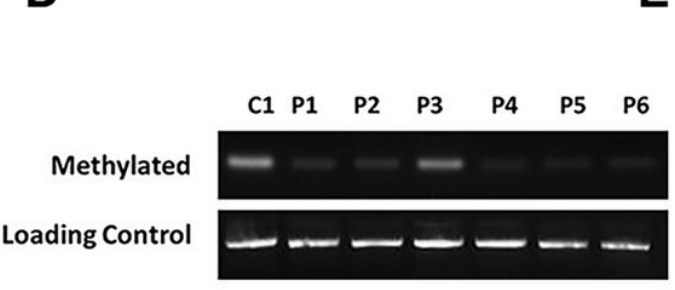

E
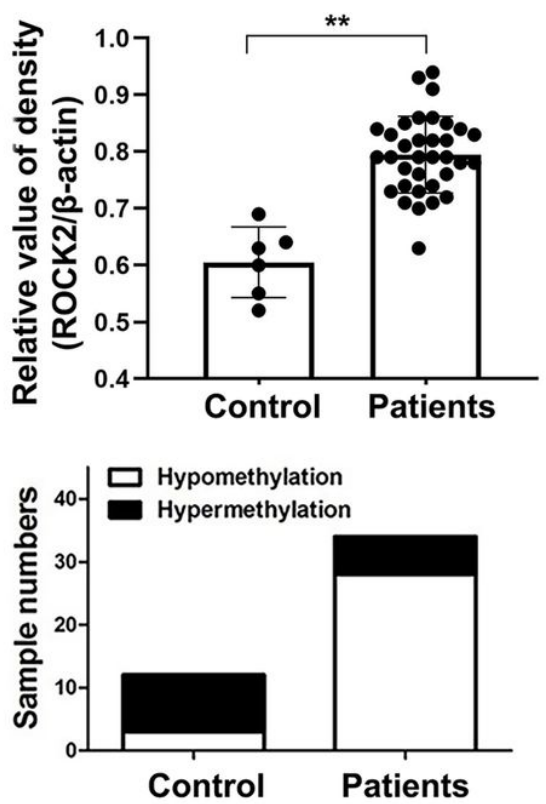

Figure 2

Elevated hippocampal ROCK2 expression by the hypomethylation of ROCK2 promoter. (A) Patient 3's pre-operative assessment and surgical resection specimens: coronal FLAIR-MRI of the head showed abnormal high signal intensity in the left hippocampus (left), FDG-PET revealed hypometabolic lesions in the left mesial temporal lobe (middle), interictal electroencephalography showed positive sharp slow waves in the left mesial temporal region (below), and the anterior temporal lobe, hippocampus and amygdala were immediately stored in the epilepsy brain bank after surgical resection (right). (B) Representative western blot assays of ROCK2 expression in the hippocampus of controls and patients with TLE. (C) Comparison of the Western blot intensity ratios of ROCK2/ $\beta$-actin in the hippocampus of controls $(n=6)$ and patients with TLE ( $n=34)$. (D) Representative methylation status of the ROCK2 promoter region in the hippocampus of controls and patients with TLE. (E) Statistical graphs show the comparison of the hypermethylation of ROCK2 promoter in the hippocampus of controls $(n=12)$ and patients with TLE ( $n=34)$. The experiments were repeated 3 times. $\mathrm{P}$ patients with epilepsy, C control. Data were analyzed using unpaired $\mathrm{t}$-tests. ${ }^{\star \star} \mathrm{P}<0.01$. 
A

$C$ 1d 3d 5d 7d 15d 30d 60d

ROCK2

$-\infty-\infty-$

$\beta$-actin
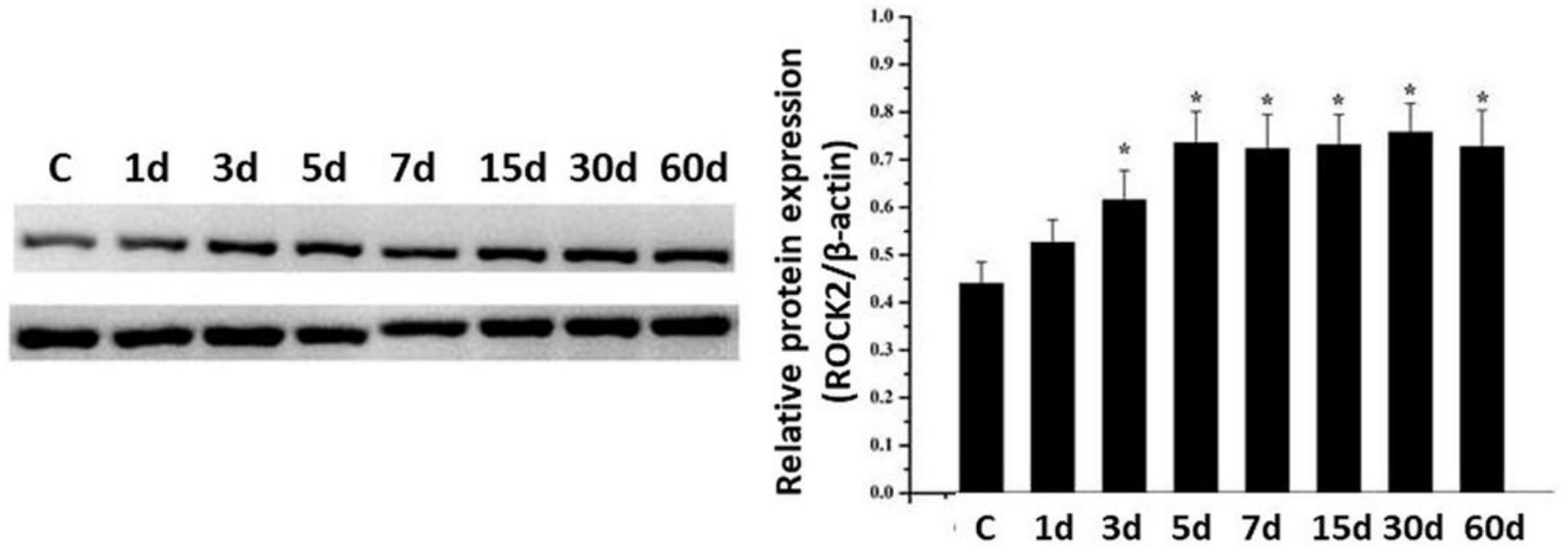

C

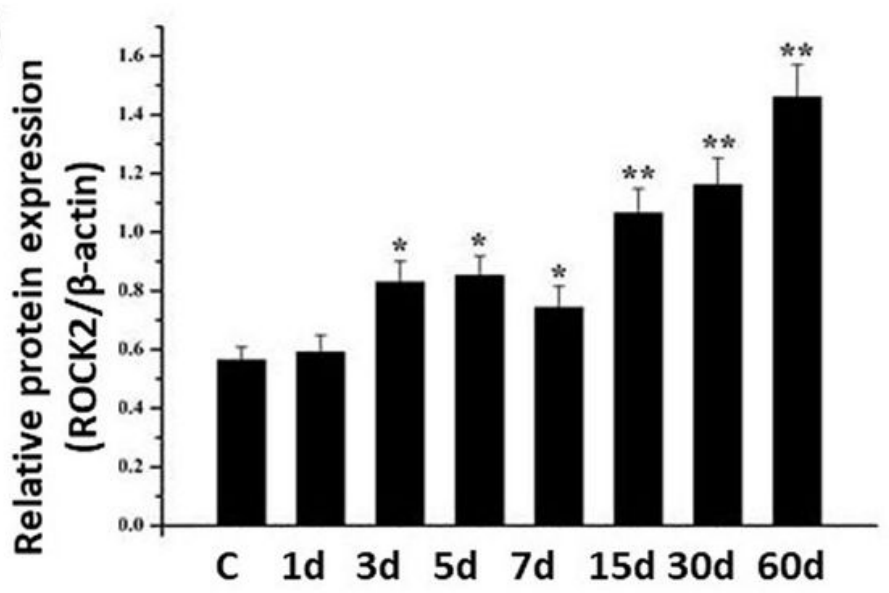

Figure 3

Increased ROCK2 Expression in the temporal cortex and hippocampus of LiCl-Pilo epilepsy Rats. (A, C) Representative western blot assays of ROCK2 expression in the temporal cortex and hippocampus at different post-seizure time points. (B, D) Compared with the control group, the expression of ROCK2 in temporal cortex and hippocampus of PILO group increased significantly from the third day after status epilepticus. The experiments were repeated 3 times with 3 rats in each group. Data were analyzed using one-way ANOVA and Dunnett's multiple comparisons test. $\star P<0.05, * * P<0.01$. 
A

$C$ 1d 3d 5d 7d 15d 30d 60d

ROCK2

$-\infty-\infty-$

$\beta$-actin
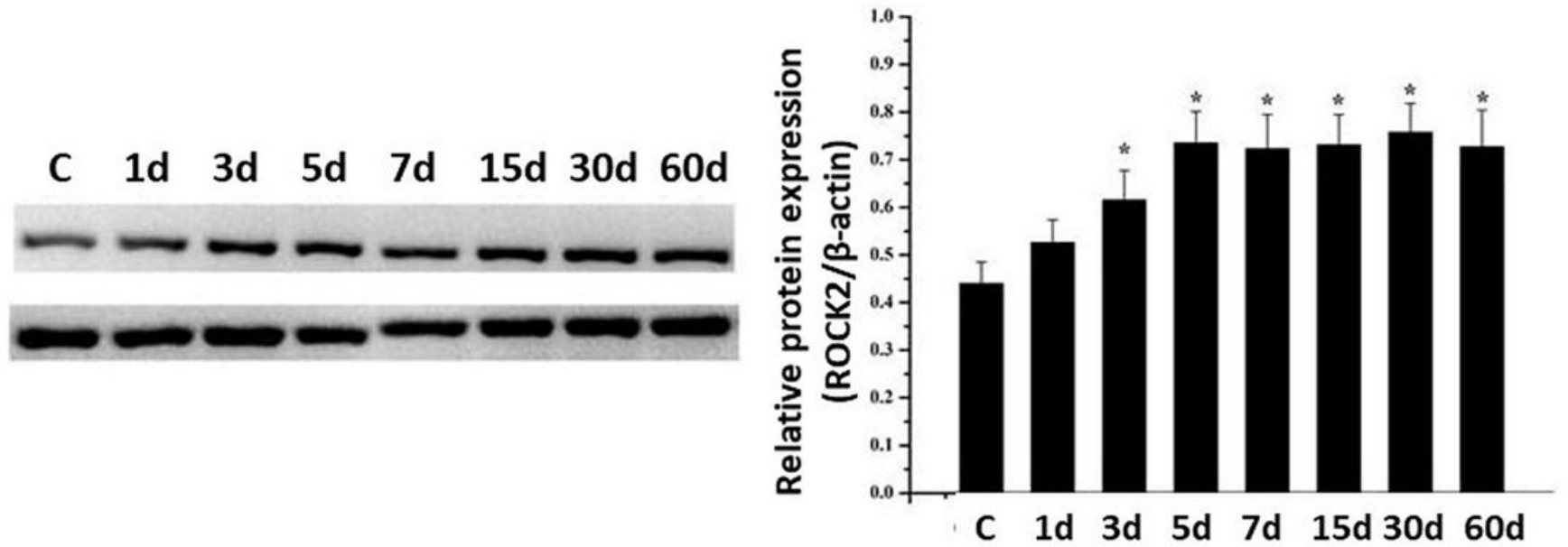

C

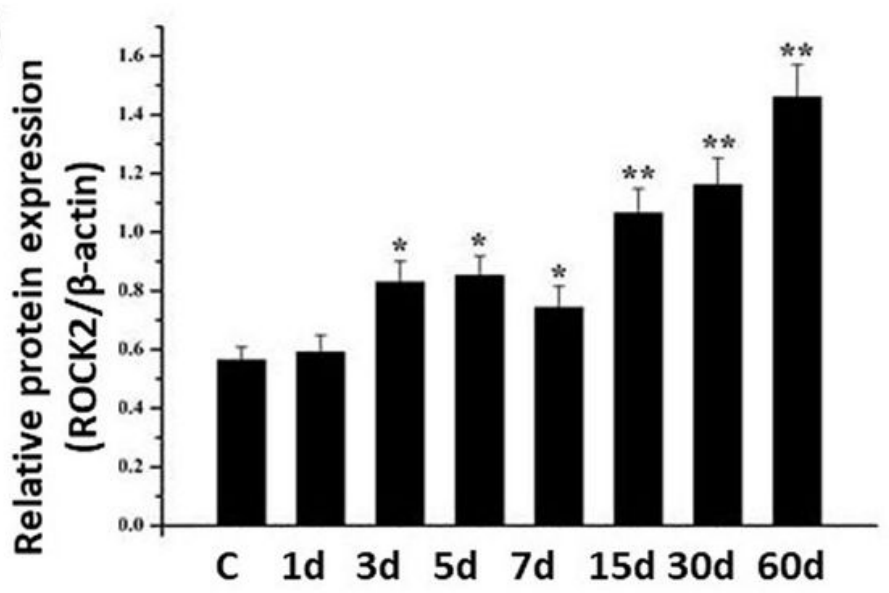

Figure 3

Increased ROCK2 Expression in the temporal cortex and hippocampus of LiCl-Pilo epilepsy Rats. (A, C) Representative western blot assays of ROCK2 expression in the temporal cortex and hippocampus at different post-seizure time points. (B, D) Compared with the control group, the expression of ROCK2 in temporal cortex and hippocampus of PILO group increased significantly from the third day after status epilepticus. The experiments were repeated 3 times with 3 rats in each group. Data were analyzed using one-way ANOVA and Dunnett's multiple comparisons test. $\star P<0.05, * * P<0.01$. 

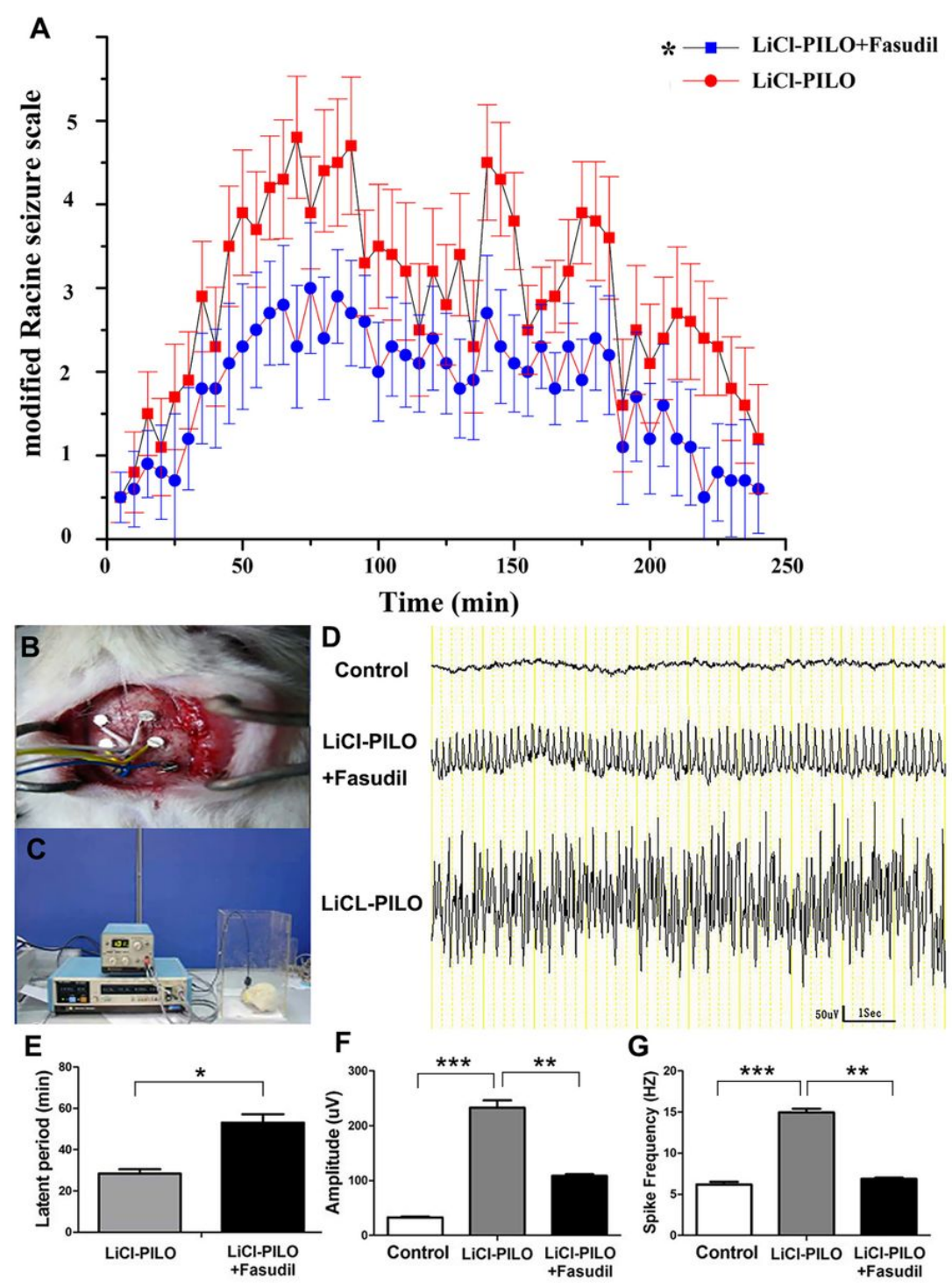

\section{Figure 4}

Fasudil reduces epileptic seizures in LiCl-Pilo epilepsy Rats. (A) PILO-induced status epilepticus was scored every 5 minutes for up to $4 \mathrm{~h}$ according to a modified Racine scale. Average scores in the Fasudil treatment group were lower than the PILO group. (B, C) The location of intracranial electrodes and its monitoring environment in LiCl-Pilo epileptic rats. (D) The representative hippocampal EEG traces at $1 \mathrm{~h}$ after pilocarpine administration were showed. (E) The latency of GTCS in the Fasudil treatment group tends to be longer than in the PILO group. (F, G) Compared with PILO group, the spike amplitude and frequency of hippocampal EEG were significantly attenuated in Fasudil treatment group. 

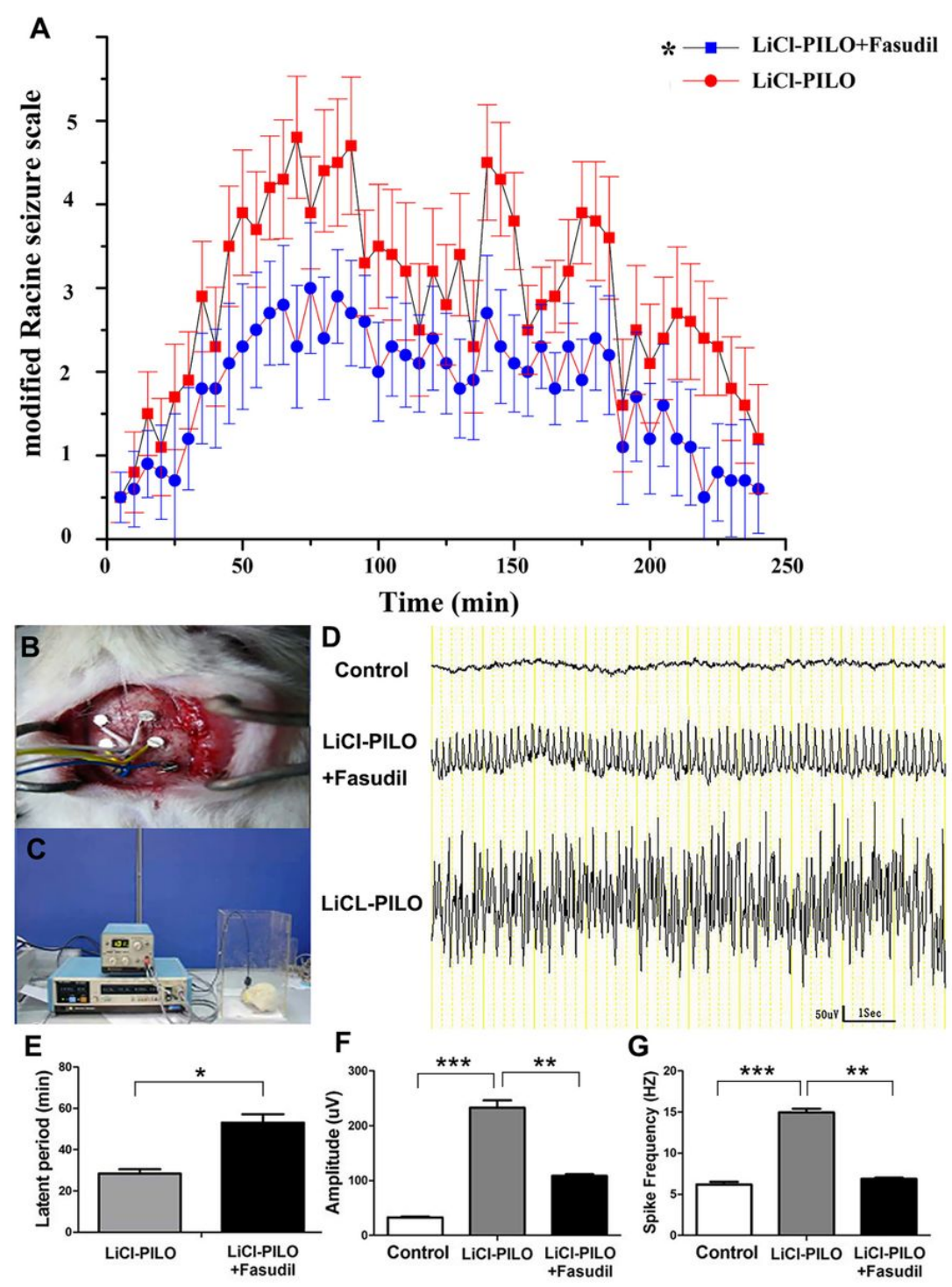

\section{Figure 4}

Fasudil reduces epileptic seizures in LiCl-Pilo epilepsy Rats. (A) PILO-induced status epilepticus was scored every 5 minutes for up to $4 \mathrm{~h}$ according to a modified Racine scale. Average scores in the Fasudil treatment group were lower than the PILO group. (B, C) The location of intracranial electrodes and its monitoring environment in LiCl-Pilo epileptic rats. (D) The representative hippocampal EEG traces at $1 \mathrm{~h}$ after pilocarpine administration were showed. (E) The latency of GTCS in the Fasudil treatment group tends to be longer than in the PILO group. (F, G) Compared with PILO group, the spike amplitude and frequency of hippocampal EEG were significantly attenuated in Fasudil treatment group. 


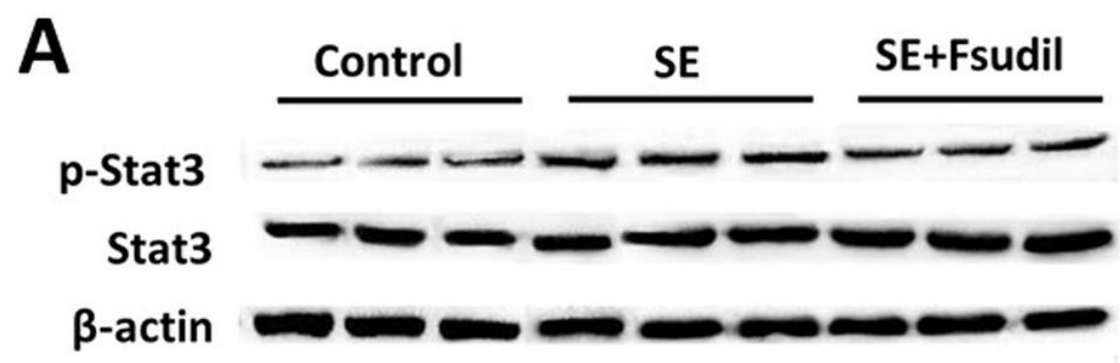

B
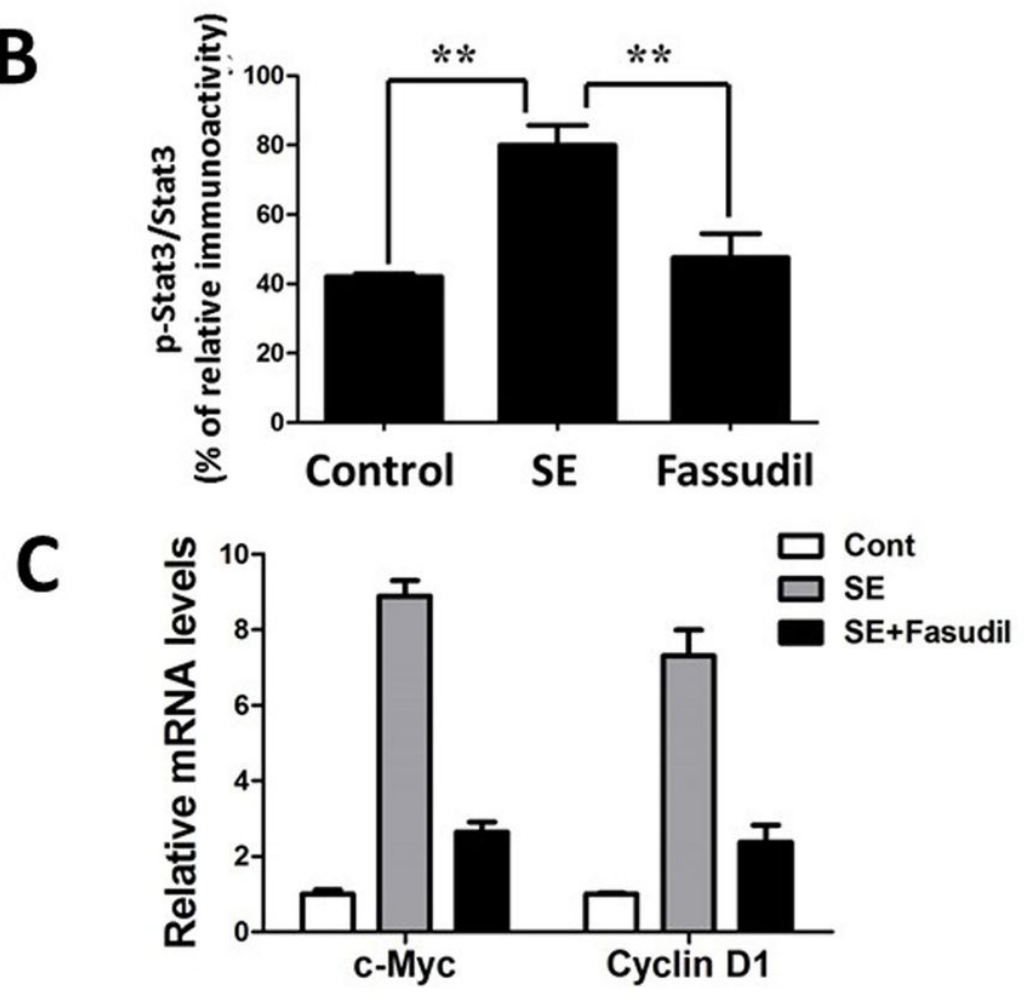

Figure 5

ROCK2 activates Stat3 pathway in Pilocarpine-Treated Epileptic Rats. (A-B) p-Stat3, Stat3 and $\beta$-actin protein levels were determined (A) and quantified in the brain tissues of rats in indicated group. (C) c-Myc and Cyclin D1 mRNA levels were determined in the brain tissues of rats in indicated group. Data are expressed as means \pm SD $(n=3)$. 


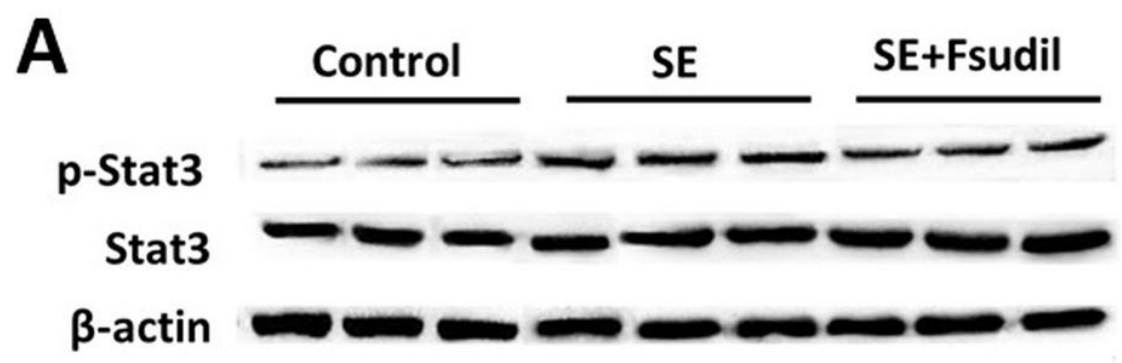

B
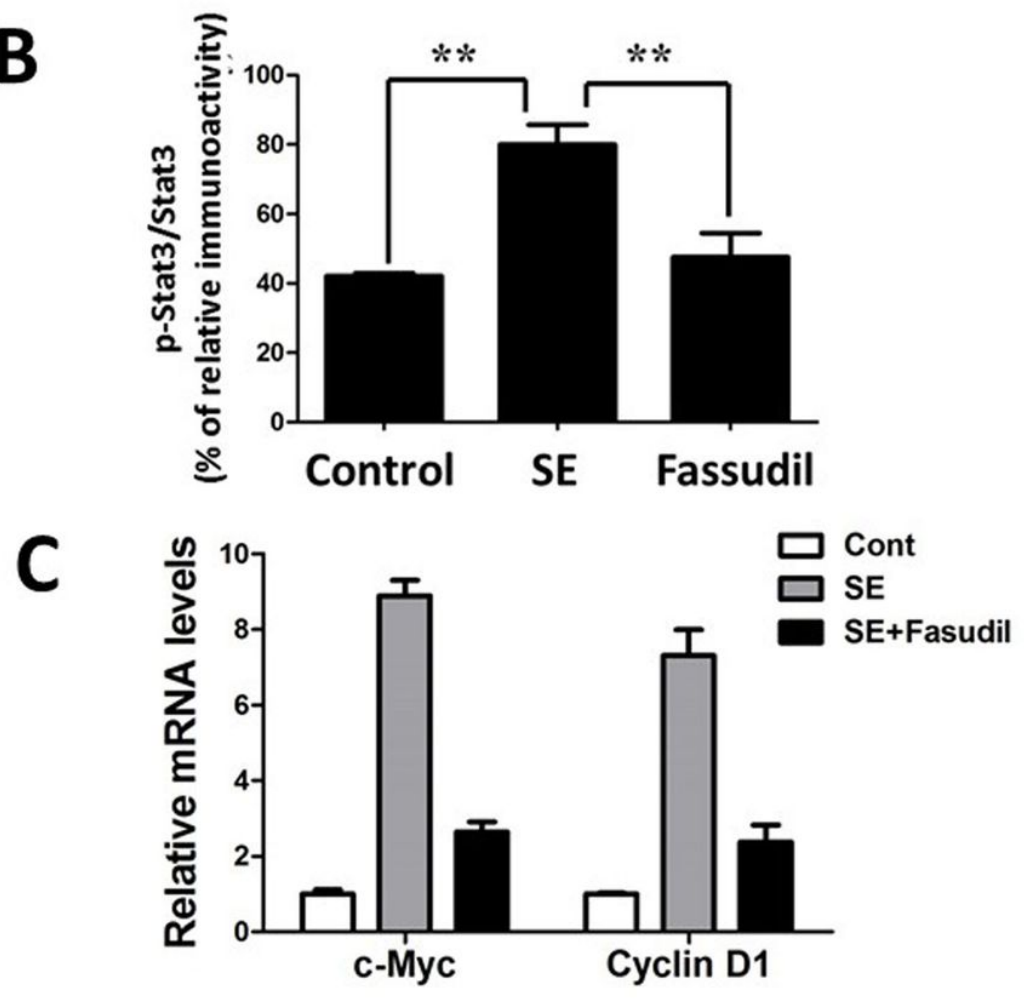

Figure 5

ROCK2 activates Stat3 pathway in Pilocarpine-Treated Epileptic Rats. (A-B) p-Stat3, Stat3 and $\beta$-actin protein levels were determined (A) and quantified in the brain tissues of rats in indicated group. (C) c-Myc and Cyclin D1 mRNA levels were determined in the brain tissues of rats in indicated group. Data are expressed as means \pm SD $(n=3)$. 


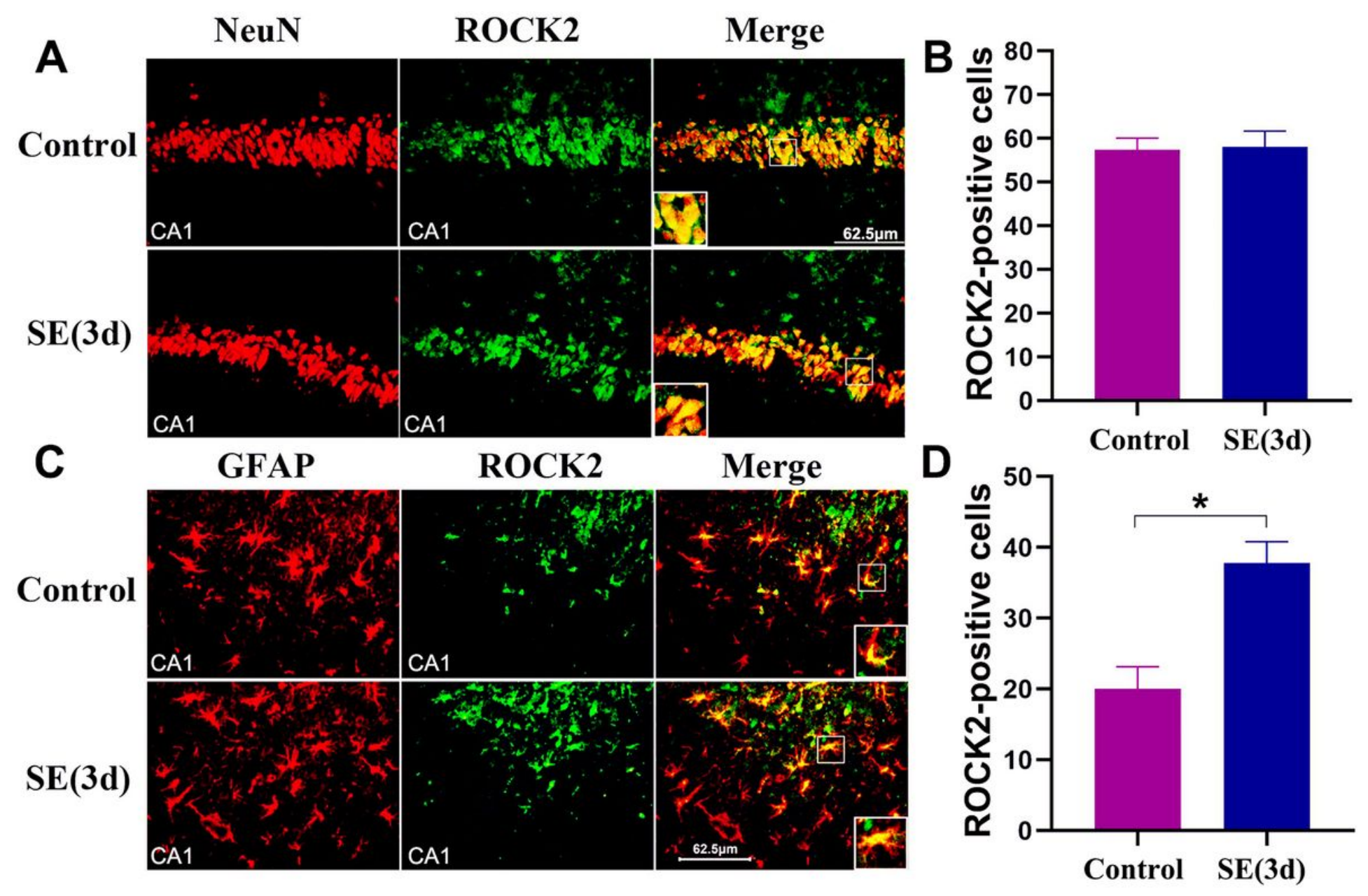

Figure 6

Cellular localization of ROCK2 in the LiCl-Pilo epilepsy rats' CA1 regions. Double immunofluorescence labelling for ROCK2 in the epileptic rats' CA1 regions during acute post-seizure phases. (A) Expression of ROCK2 and the neuronal marker NeuN in the CA1 regions. (C) Expression of ROCK2 and the glial marker GFAP in in the CA1 regions. (B, D) From the 3th day after the status epilepticus, the number of ROCK2-positive astrocytes was significantly higher than that of the control group, while the expression of ROCK2-positive neurons was not changed. * $P<0.05$ by Student's $t$ test compared to control ( $n=6$ per group). Scale bars $=62.5 \mu \mathrm{m}$. 


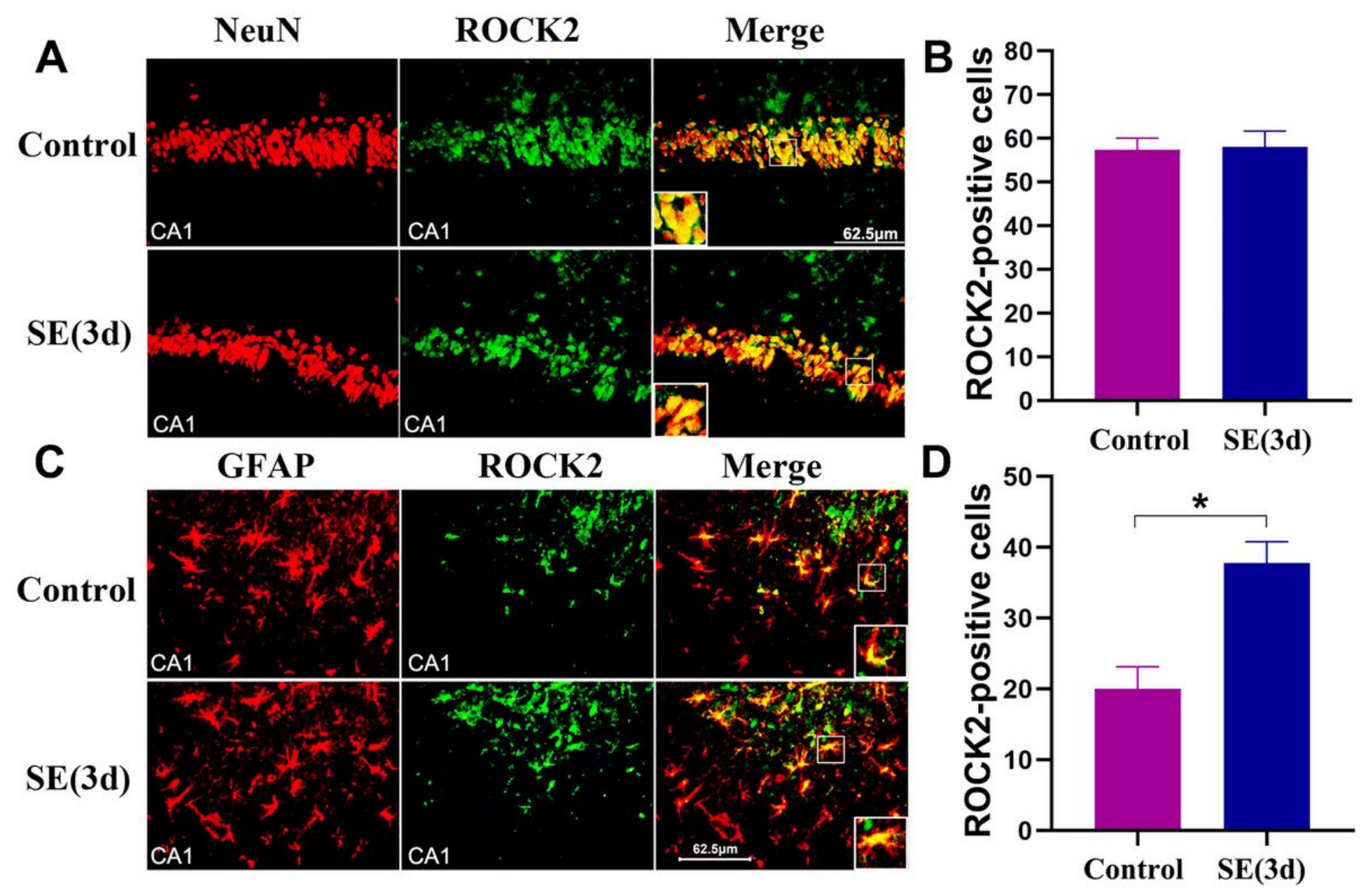

Figure 6

Cellular localization of ROCK2 in the LiCl-Pilo epilepsy rats' CA1 regions. Double immunofluorescence labelling for ROCK2 in the epileptic rats' CA1 regions during acute post-seizure phases. (A) Expression of ROCK2 and the neuronal marker NeuN in the CA1 regions. (C) Expression of ROCK2 and the glial marker GFAP in in the CA1 regions. (B, D) From the 3th day after the status epilepticus, the number of ROCK2-positive astrocytes was significantly higher than that of the control group, while the expression of ROCK2-positive neurons was not changed. * $P<0.05$ by Student's $t$ test compared to control ( $n=6$ per group). Scale bars $=62.5 \mu \mathrm{m}$. 


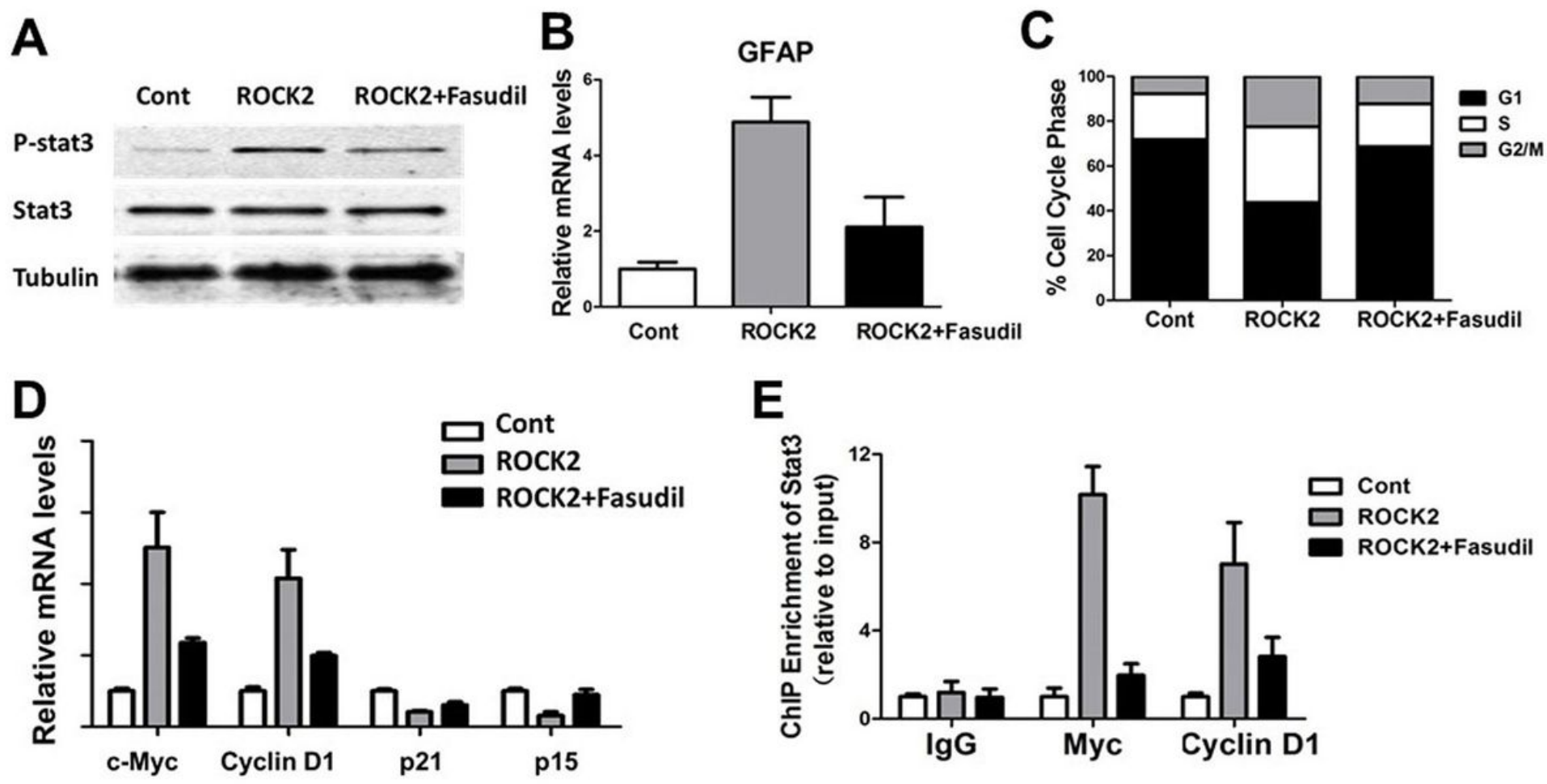

Figure 7

ROCK2 induces epileptogenesis by activating astrocyte cell cycle progression via Stat3 pathway. (A-B) Astrocytes were infected with ROCK2 overexpression lentivirus, and then treated with $5 \mu \mathrm{M}$ Fasudil for $24 \mathrm{~h}$. (A) The indicated protein levels (A), GFAP mRNA levels (B), cell cycle progression (C) were determined in indicated groups, respectively. (D-E) Stat3 target gene mRNA levels (D) and Stat3 enrichments on Myc or Cyclin D1 promoter were determined respectively. Data are expressed as means $\pm \operatorname{SD}(n=3)$.
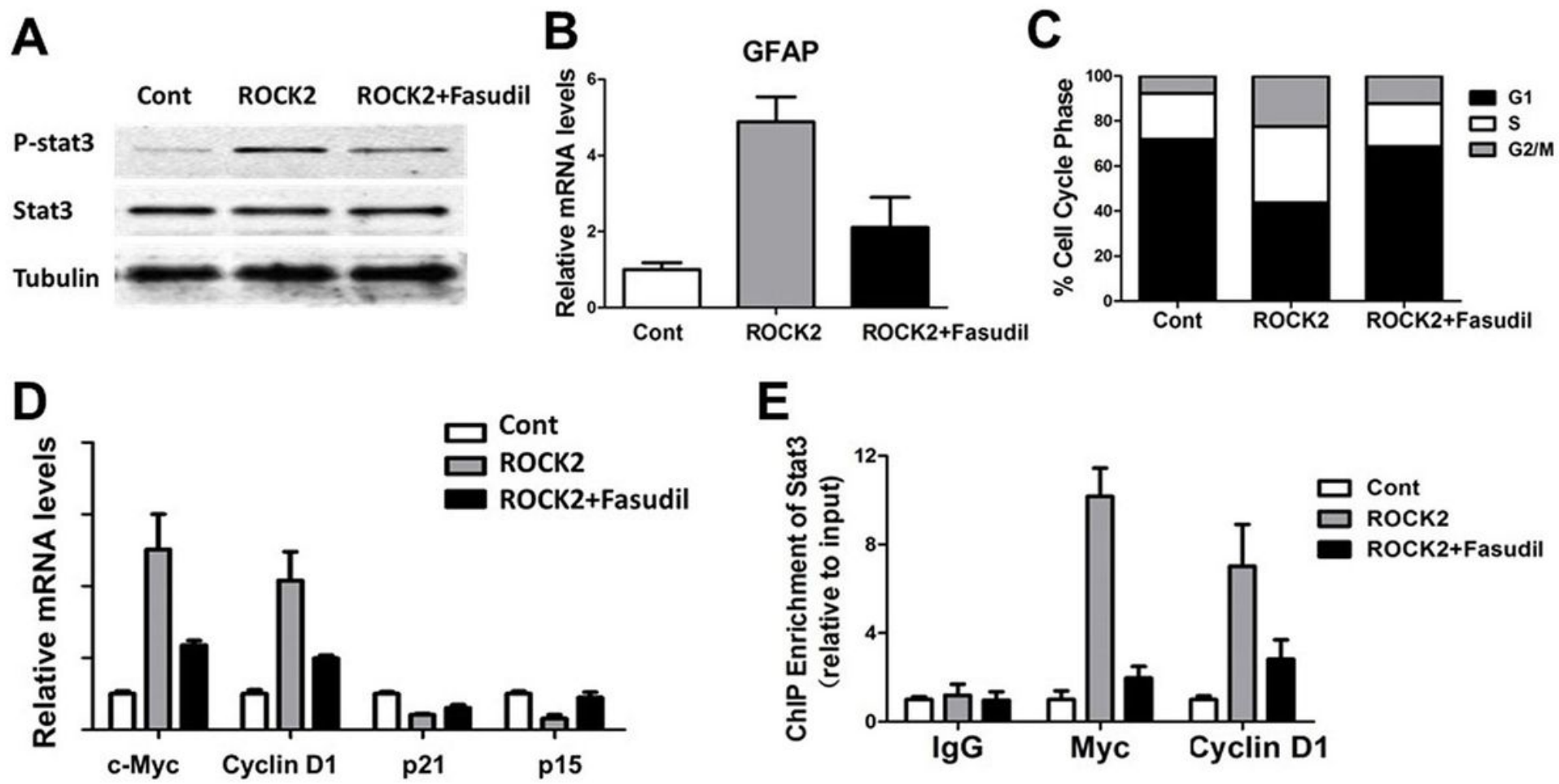

Figure 7 
ROCK2 induces epileptogenesis by activating astrocyte cell cycle progression via Stat3 pathway. (A-B) Astrocytes were infected with ROCK2 overexpression lentivirus, and then treated with $5 \mu \mathrm{M}$ Fasudil for $24 \mathrm{~h}$. (A) The indicated protein levels (A), GFAP mRNA levels (B), cell cycle progression (C) were determined in indicated groups, respectively. (D-E) Stat3 target gene mRNA levels (D) and Stat3 enrichments on Myc or Cyclin D1 promoter were determined respectively. Data are expressed as means \pm SD $(n=3)$.
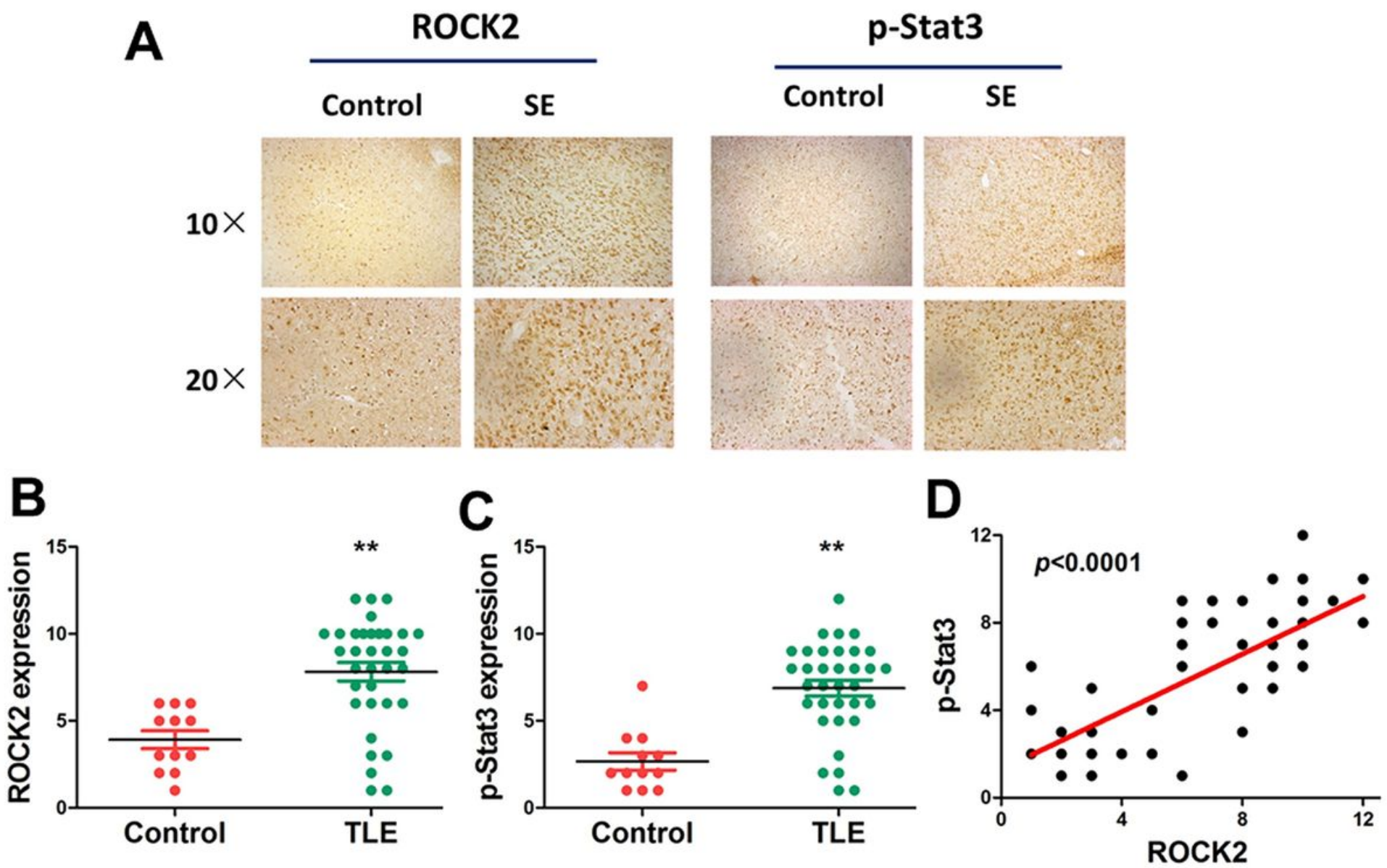

Figure 8

ROCK2 is associated with Stat3 activation in TLE patients. (A) Immunohistochemistry staining of ROCK2 and p-Stat3 in normal human and TLE patient tissues. $(B, C)$ Relative expression level of ROCK2 or p-Stat3 in human tissue samples. Student's t-test was applied for statistical analyses. **, $\mathrm{P}<0.01$ (D) Positive correlation between ROCK2 and p-Stat3 expressions with linear regression and Pearson's correlation significance ( $P$ $<0.0001$, ANOVA test). 

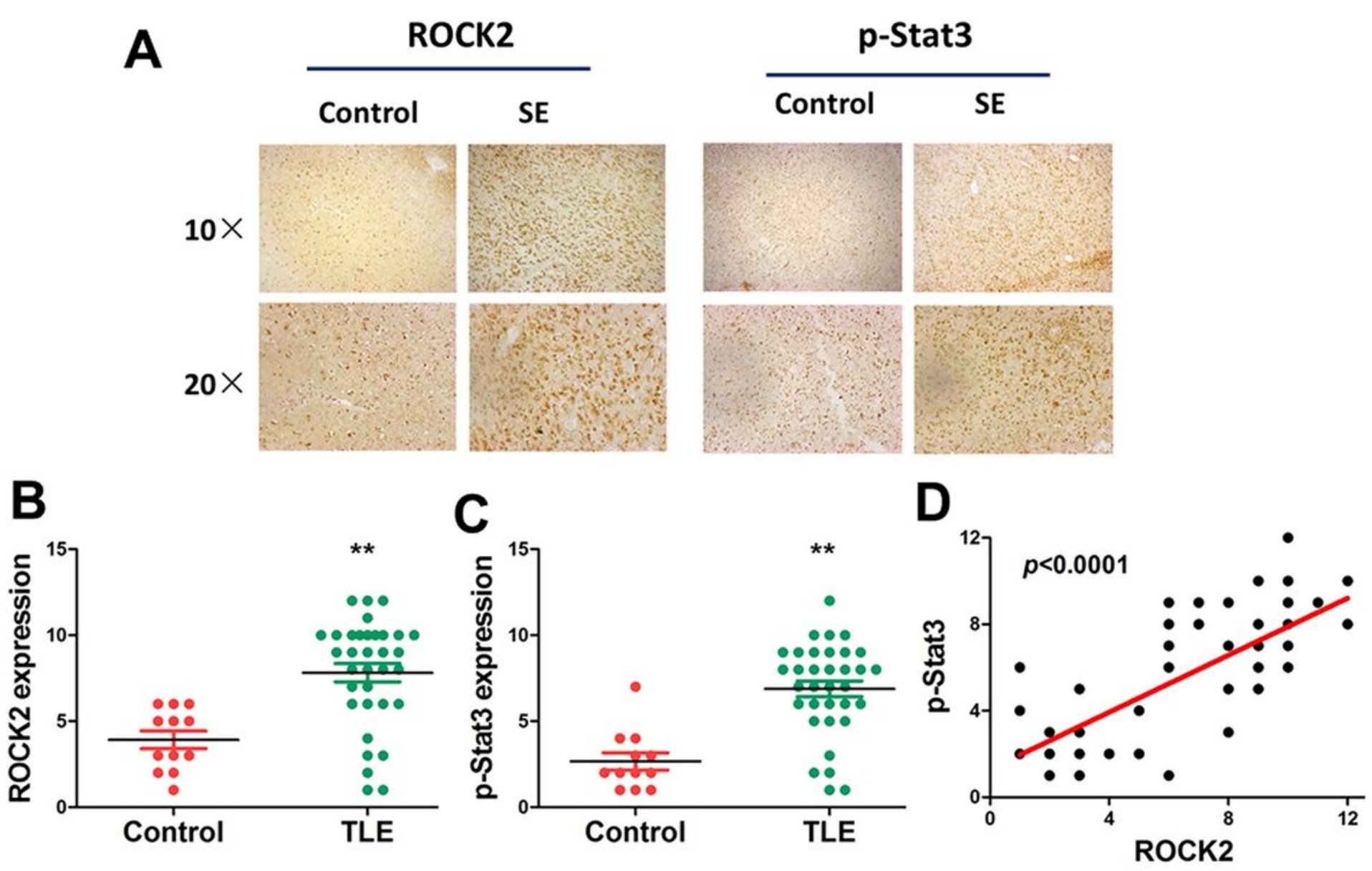

Figure 8

ROCK2 is associated with Stat3 activation in TLE patients. (A) Immunohistochemistry staining of ROCK2 and p-Stat3 in normal human and TLE patient tissues. $(B, C)$ Relative expression level of ROCK2 or p-Stat3 in human tissue samples. Student's t-test was applied for statistical analyses. **, $P<0.01$ (D) Positive correlation between ROCK2 and $p$-Stat3 expressions with linear regression and Pearson's correlation significance ( $P$ $<0.0001$, ANOVA test). 